\title{
DIE LEIPZIGER ÜBERSETZUNGSWISSENSCHAFTLICHE SCHULE, DAS TSCHECHISCHE UND PERZEPTIVE TRANSLATOLOGIE AVANT LA LETTRE
}

\author{
CARSTEN SINNER
}

\begin{abstract}
The Leipzig School of Translation, Czech, and Perceptive Translation and Interpreting Studies avant la lettre

Following an overview of both the history of translator and interpreter training in Leipzig and of the Leipzig School of translation, this article first discusses the gaps and deficiencies in the reception of the Leipzig School of translation before showing that this criticism was often unjustified and due to a lack of knowledge of the texts produced by the School in question. In the following, two aspects are examined in more detail: the question of the understanding of Sprachmittlung 'linguistic mediation' and Translation 'translation and interpreting', i.e. a terminological and conceptual view that reveals the apparently inadequate understanding of key texts of the Leipzig School by many of the authors who criticised it; finally, an exemplary examination of one of the key texts (Jäger 1975) allows for its innovative capacity to be brought to light.
\end{abstract}

Keywords: T\&I; Leipzig School of Translation; Czech; German; Perceptive Translation Studies; concepts; terminology

\section{Einleitung}

Die Ausbildung im Bereich Übersetzung und Dolmetschen in Leipzig hat eine lange Tradition. Dies ist bis heute so: Das Institut für Angewandte Linguistik und Translatologie und das Institut für Slavistik der Universität Leipzig arbeiten seit vielen Jahren eng zusammen, etwa um den 2014 eingerichteten binationalen Studiengang Bachelor Interkulturelle Kommunikation und Translation Tschechisch-Deutsch in Kooperation mit dem Institut für Translatologie der Karls-Universität (Prag) zu ermöglichen. Diese Zusammenarbeit hat selbst wiederum eine lange Vorgeschichte, die in die Anfänge der universitären Ausbildung im Bereich Dolmetschen und Übersetzen in Leipzig zurückreicht.

Insbesondere im Kontext der theoretischen bzw. wissenschaftlichen Auseinandersetzung mit der Sprachmittlung und somit im Zusammenhang der Herausbildung der heute als Leipziger übersetzungswissenschaftliche Schule bekannten Ausrichtung kommt dem Sprachenpaar Deutsch und Tschechisch schon seit frühen Zeiten eine besondere Rolle zu. 
Der Slawist und allgemeine Linguist Rudolf Růžička hatte enge Kontakte in die Tschechoslowakei, etwa zu den Prager Kollegen Petr Sgall und Karel Hausenblas, und dank eines alten Universitätsvertrages mit der Karls-Universität gab es lange Jahre einen vielfältigen Austausch von Studierenden, Lehrkräften und Wissenschaftlern und Wissenschaftlerinnen, und zwar nicht nur in der Bohemistik und Germanistik, sondern beispielsweise auch zwischen der DDR-Romanistik (etwa dem Romanisten Gerd Wotjak) und tschechischen Romanisten und Romanistinnen wie Josef Dubský und Jana Králová vom Prager Dolmetscherinstitut. Auch beherrschten mit Gert Jäger und Otto Kade zwei der zentralen Figuren der Leipziger Schule Tschechisch auf sehr gutem oder muttersprachlichem Niveau. Jäger war mit Deutsch und Tschechisch aufgewachsen, hatte u.a. Tschechisch studiert und baute in Leipzig eine sehr leistungsstarke Abteilung für Tschechisch, Slowakisch und Polnisch mit auch bohemistisch arbeitenden Kollegen und Kolleginnen wie Bernd Koenitz, Dietrich Müller und Richard Rothenhagen auf und sorgte dafür, dass viele der Mitarbeiter und Mitarbeiterinnen in diesem Bereich zum Übersetzen und zum Sprachvergleich - hierzu organisierte er selbst mehrere internationale Tagungen - promovierten.

Die Sprachmittlung zwischen dem Tschechischen und Deutschen und die darauf beruhenden theoriebegründenden Überlegungen zum Übersetzen als Prozess waren also in der Herausbildung der Ansätze der Leipziger Schule überaus relevant. So erstaunt es umgekehrt auch nicht, dass in Tschechien die Leipziger Schule bis heute in der Translatologie von Relevanz und entsprechend präsent ist (s. beispielsweise Hrdinová 2018 und im Druck), wenngleich Heidemarie Salevsky (in einer E-Mail an Benešová, s. Benešová 2019, 19) der Auffassung ist, dass der Austausch zwischen Karl-Marx-Universität und Karls-Universität gering gewesen sei. Benešová $(2019,19)$ weist auf den Umstand hin, dass Kade in seinen Werken unter anderem auf die tschechischen Linguisten Miloš Dokulil, Oldřich Leška, Petr Sgall und Bohumil Palek und insbesondere auch auf das Werk Umění překladu ,Die Kunst des Übersetzens' (in deutscher Sprache 1969 mit dem Titel Literarische Übersetzung. Theorie einer Kunstgattung erschienen) von Jiří Levý in ihrer tschechischen Version von 1963 hinweist. Levýs Werk sieht Kade als wichtigen Beitrag zur Theorie der literarisch-künstlerischen Übersetzung an und es hat, so Benešová $(2019,19)$, im Werk Kades deutliche Spuren hinterlassen.

Die Auseinandersetzung mit der Sprachmittlung - und entsprechend auch der Sprachmittlung zwischen Deutsch und Tschechisch - in der Leipziger Ausbildungsstätte für Dolmetschen und Übersetzen manifestierte sich in sehr innovativen und zukunftsweisenden, vor allem, aber nicht nur stark linguistisch geprägten Ansätzen, welche die wissenschaftliche Auseinandersetzung mit der Sprachmittlung damals wie heute enorm befruchten konnten und ihrer Zeit in manchen Aspekten weit voraus waren.

Diese Leistungen der Leipziger Schule wurden jedoch lange ignoriert, ihre Ansätze mitunter einseitig und ohne Quellenkenntnis für überholt, engstirnig, zu linguistisch usw. kritisiert und sogar ihre Terminologie mit fehlerhaften Daten dargelegt oder gänzlich fehlinterpretierend dargestellt.

Dieser Beitrag möchte darum, nach einem kurzen Überblick über die Geschichte der Ausbildung von Sprachmittlern und Sprachmittlerinnen in Leipzig, die besondere Innovationskraft der Leipziger Schule anhand der Auseinandersetzung mit der disziplinbezogenen Terminologie illustrieren und zeigen, dass eine Kritik an der Herangehensweise der Leipziger Schule als zu linguistisch und ausschließlich ausgangstextbezogen nicht 
gerechtfertigt war bzw. ist. Anhand einer Analyse eines Beispieles aus dem Sprachenpaar Deutsch-Tschechisch, konkret ein von Jäger (1975) betrachtetes tschechisch-deutsches Übersetzungsproblem, ${ }^{1}$ soll dann aufgezeigt werden, dass die Ansätze der Leipziger übersetzungswissenschaftlichen Schule eine in der Wissenschaftsgeschichte gerne übersehene innovative Sicht auf Übersetzung hatten und bis heute an Relevanz und Inspirationskraft nichts verloren haben. In diesem Kontext soll auch die herausragende Bedeutung der Untersuchung der Sprachmittlung anhand des Sprachenpaares Deutsch-Tschechisch für die translationswissenschaftliche Theorienbildung in Leipzig verdeutlicht werden. Abschließend soll in diesem Zusammenhang auf Desiderata der Forschung zur Rolle des Tschechischen in der Theorienbildung und translatologischen Arbeit der Leipziger Schule allgemein hingewiesen werden.

\section{Die Sprachmittlungsausbildung in Leipzig und die Leipziger übersetzungswissenschaftliche Schule}

Hervorgegangen ist die Präsenz der universitären Ausbildung im Bereich Translation an der Universität Leipzig aus einem Dolmetscherinstitut an der Handelsschule Leipzig, wo man 1937 mit vierzig Studierenden den Lehrbetrieb aufgenommen hatte. ${ }^{2}$ Dieses Institut wurde nach dem Krieg, 1946, aufgrund der Kriegszerstörungen in die Wirtschaftsund Sozialwissenschaftliche Fakultät der Universität Leipzig eingegliedert, dann aber fast umgehend von der russischen Militärverwaltung geschlossen und kurz danach in die Hände der Leipziger Fremdsprachenschule gelegt, wo im Dezember 1950 Otto Kade, der spätere Begründer der Leipziger Übersetzungswissenschaft, als pädagogischer Leiter eingesetzt wurde.

Die Ausbildung umfasste damals die Sprachen Englisch, Französisch und Russisch, 1951 wurden die Sprachen Polnisch, Tschechisch und (zumindest zeitweilig) Chinesisch in die Ausbildung eingeführt.

1953 wurde die Ausbildung von Übersetzung und Dolmetschen weiter aufgewertet, die Fremdsprachenschule wurde als „Fachrichtung Dolmetscher und Übersetzer“ in das neu gegründete Pädagogische Institut Leipzig eingegliedert, wodurch die Ausbildung wieder Hochschulcharakter erhielt. 1956 schließlich wurde die „Fachrichtung Dolmetscher und Übersetzer“ als selbständiges Institut (Dolmetscher-Institut) an die damalige Karl-Marx-Universität überführt.

1962 wurde Albrecht Neubert als Professor für Englische Übersetzungswissenschaft berufen. Zusammen mit dem Russisten Otto Kade und dem Westslawisten Gert Jäger begründete er die - später von außerhalb so bezeichnete - Leipziger übersetzungswissenschaftliche Schule, die ihre Grundpositionen auf fünf Konferenzen zwischen 1965 und 1991 unter dem Leitthema „Grundfragen der Übersetzungswissenschaft“ vortrug.

Diese Konferenz zu den Grundfragen der Übersetzungswissenschaft gibt es, mit leicht veränderter Bezeichnung, bis in die Gegenwart. Die 2017 am Institut für Angewandte

Zur Unterscheidung von Problem und Schwierigkeit in der Übersetzung s. Nord (1987).

2 Die Darstellung der Geschichte der Ausbildung im Bereich Sprachmittlung folgt den Ausführungen von Lutz (2008 und o. J.) sowie unterschiedlichen Texten und persönlichen Aussagen von Gerd Wotjak zur Leipziger Schule. 
Linguistik und Translatologie (IALT) abgehaltene 10. Leipziger Internationale Konferenz zu Grundfragen der Translatologie mit über 200 Teilnehmenden steht für die Kontinuität der translatologischen Forschung am IALT und die wichtige Rolle des Instituts in der internationalen Forschungslandschaft. Vor allem ist diese traditionsreiche Konferenz ein Symbol für die besondere Bedeutung der Präsenz der Translation an dieser Universität und die Relevanz der Leipziger übersetzungswissenschaftlichen Schule, die, wie zu zeigen sein wird, immer wieder auch mit dem Tschechischen zu tun hat.

Die Namen, Kade, Jäger, Neubert, später dann auch Heide Schmidt und Gerd Wotjak, werden in der Translatologie einen wichtigen Platz einnehmen. Auf den Westslavisten Gerd Jäger ist später zurückzukommen.

Die Ausbildung im Bereich Übersetzung an der ehemaligen Karl-Marx-Universität Leipzig war früher zusammen mit der Ausbildung in Sprachwissenschaft und Sprachen an einer Einrichtung vereint. Das hat sich nach der politischen Wende mit der Neuorganisation der Universität geändert. In den Jahren nach der Wiedervereinigung wurden die translatologischen Forschungen am Institut für Sprach- und Übersetzungswissenschaft, das 1999 in Institut für Angewandte Linguistik und Translatologie (IALT) umbenannt wurde, trotz des Ausscheidens einiger führender Vertreter konsequent weitergeführt. Im neuen Jahrtausend wurden die translatologische Forschung und Lehre am IALT um Schwerpunkte im Bereich der Translation in der Intersektion mit Varietätenlinguistik, Kognitionswissenschaft, Perzeptionsanalyse, Minderheitensprachen und Sprachpolitik sowie etwa um Studien zur Audiovisuellen Übersetzung oder auch der Historischen Translatologie - zu nennen sind hier auch die wissenschaftliche Auseinandersetzung mit dem Erbe und der Kontinuität der Leipziger übersetzungswissenschaftlichen Schule oder die Arbeit im Bereich der Oral History der Translatologie - erweitert.

\section{Berechtigte Kritik oder fehlende Einblicke? Zur lücken- und mangelhaften Rezeption der Leipziger übersetzungswissenschaftlichen Schule}

Nachdem sie in der internationalen Translatologie bzw. Translation Studies viele Jahre lang weitgehend übergangen, totgeschwiegen oder mit einigen wenigen Seitenhieben als zu linguistisch, in der generativen Linguistik verhaftet, aber durch kulturwissenschaftliche Positionen „überwunden“ dargestellt worden ist (s. Jung et al. 2013, Sinner 2016, Cañuelo et al. 2019), wurde in jüngeren Texten zur Translatologie fast mehr über die Gründe für die mangelhafte Rezeption der Leipziger übersetzungswissenschaftlichen Schule außerhalb der DDR und der mit ihr wirtschaftlich und ideologisch nahestehenden Staaten geschrieben als über die grundlegenden wissenschaftlichen Positionen und Sichtweisen ihrer Vertreter und Vertreterinnen oder darüber, wie sich diese Positionen in der didaktischen Herangehensweise an die Translation - ablesbar beispielsweise an den Studienplänen und inhalten der translatologischen Ausbildung in der DDR (s. Sinner 2020, im Druck) - manifestierten.

Der Umstand, dass die Leipziger Schule in Westdeutschland und in der internationalen Translatologie so stiefmütterlich behandelt wurde, wurde auf verschiedene Ursachen zurückgeführt, darunter insbesondere auf 
- das Fehlen von Übersetzungen der Schlüsseltexte der Leipziger Schule oder Publikationen ihrer wesentlichen Positionen in international „zugänglicheren“ Sprachen als Deutsch und slawische Sprachen wie Russisch oder Tschechisch;

- den Kalten Krieg, den Eisernen Vorhang und die damit verbundene, an Vorurteile oder ideologische Motive gebundene Zurückweisung von pauschal als kommunistisch oder sozialistisch angesehenen Texten;

- die fragwürdige Praxis der Arbeit mit Sekundärquellen anstelle der Lektüre der Primärtexte, also der Rezeption der Leipziger Schule über Beschreibungen in Arbeiten anderer Autoren und Autorinnen selbst dann, wenn diese auf Texte hätten zurückgreifen können, die in von ihnen gesprochene Sprachen vorlagen; ${ }^{3}$

- das bloße Desinteresse an diesen Texten trotz ihrer „physischen“ Zugänglichkeit, wie sie Wotjak konstatiert, der nach dem Fall der Mauer in westdeutschen Bibliotheken fundamentale Veröffentlichungen der Leipziger Schule mit nicht aufgeschnittenen Seiten - also definitiv ungelesen - vorfand (s. Snell-Hornby 2007, Jung et al. 2013, Castro Yáñez 2015, Sinner 2016);

- die Zurückweisung jeglicher als linguistisch angesehenen Herangehensweise an Übersetzung in Westdeutschland, insbesondere in den 1970er und 1980er Jahren, und zwar weniger aus wissenschaftlicher Überzeugung denn aufgrund persönlicher Beweggründe bestimmter Übersetzungswissenschaftler und Übersetzungswissenschaftlerinnen, die ein besonderes Interesse daran hatten, die Übersetzungswissenschaft als Disziplin aus der Linguistik herauszulösen, um dadurch Stellen für Fachleute der Übersetzungswissenschaft an den neu gegründeten Instituten für Übersetzungswissenschaft zu sichern (Pym 2014).

Bis heute werden die Beiträge der Leipziger Schule aus diversen, vielfach nicht annähernd gerechtfertigt erscheinenden Gründen und auf Grundlage einer minimalen Textgrundlage kritisiert, wenn man sich überhaupt die Mühe macht, die Originaltexte einzusehen. Immer wieder werden das breite Spektrum der wissenschaftlichen Auseinandersetzung mit Sprachmittlung und die mannigfaltigen Überlegungen und Vorschläge der Leipziger Schule zu Methode und Theorie der Translation auf Einzelaspekte reduziert, häufig auf ein einziges Modell bzw. sogar eine einzige Graphik dieses Modells - eine frühe Fassung des später wesentlich erweiterten Modells der zweisprachigen vermittelten Kommunikation $^{4}$ - oder auf das vermeintlich problematische Verständnis von Äquivalenz, ${ }^{5}$ und noch immer wird auf einer solch mageren Grundlage die Leipziger Schule pauschalisierend kritisiert (vgl. Wotjak 2002, 2002-2003, Cañuelo et al. 2019).

3 S. Cañuelo et al. (2019) für eine Kritik der Be- bzw. Verurteilungen von Kades Verständnis von Äquivalenz durch Albir oder Vidal Claramonte auf Grundlage der verkürzten Darstellung in Arbeiten von Snell-Hornby.

4 Besonders deutlich wird die fehlende Einsicht mancher der Kritik übenden Autoren und Autorinnen daran, dass sie sich auf Aussagen zu einer sehr frühen Fassung des Modells der zweisprachigen gemittelten Kommunikation beschränken, das in einer ersten Variante zwei Phasen aufwies, während alle späteren Versionen - wie etwa bei Jäger $(1975,28)$ - dagegen drei Phasen aufweisen.

5 Nur selten wird aufgezeigt, dass und wie sich das Verständnis der Äquivalenzbeziehungen zwischen Ausgangs- und Zieltext in der Leipziger Schule entwickelte, oder die Relation zwischen dem kommunikativen Wert und der funktionalen Äquivalenzbeziehung zwischen Ausgangs- und Zieltext betrachtet. Siehe hierzu de la Cruz Recio (2016). 
Zu den Kritikpunkten, die, wie in Sinner (2016) aufgezeigt wurde, nicht oder nur bedingt gerechtfertigt sind oder mit einem falschen Verständnis der Ausgangstexte erklärt werden müssen, gehören unter anderem Kritik

- an der Idee von Übersetzungsnormen, die man als präskriptiv interpretierte und zurückwies;

- am Terminus des Kodierungswechsels bzw. der Umkodierung, verstanden als „Umschlüsselung eines gegebenen Textes aus dem Kode-System AS[=Ausgangssprache] in das Kode-System ZS [Zielsprache]“ (Kade 1968, 54);

- an der vermeintlich zu eng gefassten Definition von Übersetzung, welche etwa die als kommunikativ heterovalent angesehene Textbearbeitung und die bewusste Manipulation von Texten ausschließt, womit die Leipziger Schule diese nicht zu den Hauptanliegen der Translatologie zählt (s. Bolaños Cuéllar 2008, 21 als Beispiel für eine solche Kritik). Tatsächlich ging die Leipziger Schule von deskriptiven Übersetzungsnormen aus und verstand unter Norm oder Regel das, was die übersetzenden Personen in der Praxis tatsächlich normalerweise bzw. regelmäßig tun bzw. was zur Erreichung eines Zieles konkret unternommen werden muss (s. Neubert 1965, vor allem 1965, 89).

Die Kritik wies den Ausdruck Kodierungswechsel bzw. Umkodierung zurück, weil er vermeintlich das Bild einer zu sehr auf die mechanische Umsetzung von Elementen reduzierte Handlung zeichne. Dies galt als abwegig und wurde abgelehnt, weil man eben nicht „einfach“ nur ein Element der einen Sprache durch ein Element der anderen Sprache austausche. Mit ihrer Sicht stellen sich die Kritiker und Kritikerinnen jedoch selbst als unzureichend in die Materie eingearbeitet bloß, denn mit dieser Haltung wird deutlich, dass sie übersehen bzw. nicht wissen, dass es sich hier um einen rein terminologischen Aspekt handelt, der in den Umständen seiner Zeit zu erklären ist, als sprachliche Konsequenz der in der Sprachwissenschaft - der „Mutterdisziplin“, aus der sich die Übersetzungswissenschaft ja herausbildete - lange Zeit üblichen Gleichsetzung von Sprache mit Code bzw. Kode. Bis heute finden sich Zeugnisse dieser terminologischen Gleichsetzung in Termini wie Code Switching bzw. Kodewechsel für den Wechsel von einer in eine andere Sprache im Rahmen einer Äußerung oder einer Reihe von Äußerungen.

Der Vorwurf, dass sich die im Kontext der Linguistik herausgebildete Leipziger Schule explizit nicht mit nicht-linguistischen und subjektiven Aspekten auseinandersetzen wollte, muss erstaunen. Bolaños Cuéllar $(2008,21)$, der der Auffassung ist, die Ansätze der Leipziger Schule benötigten „broadening of perspective to include extra-linguistic aspects" (Bolaños Cuéllar 2008, 17), schreibt beispielsweise:

The communicative nature of translation and its linguistic basis had already been incorporated into the approach developed by scholars representing this school [= the Leipzig School]. Such extra-linguistic factors as socio-historical aspects were acknowledged as issues affecting the translational communicative process. However, they were not studied scientifically, because, according to the Leipzig School's conception of what translatology should be as a science, these factors occur only once, i.e. they are not recurring events, and thus no generalization can be made concerning them. This understanding of translatology can be considered nowadays as the main shortcoming of this approach.

Es erscheint ungerecht und geradezu scheinheilig, einerseits die Vorreiterrolle der linguistischen Ansätze zu loben und ihnen gleichzeitig vorzuwerfen, dass sie sich nicht an Aspek- 
te heranwagen, für deren wissenschaftliche Erforschung man nicht ausgebildet war, für die man sich somit schlicht nicht ausreichend kompetent fühlte und die man insbesondere für nicht wissenschaftlich messbar hielt. Wie abstrus diese Kritik ist, wird deutlich, wenn man die Argumentation umkehrt: Es würde wohl niemand wagen, die ,enge Perspektive der erklärt kultur- und literaturwissenschaftlich orientierten Ansätze zu kritisieren und eine ,Erweiterung um linguistische Aspekte` zu fordern (Sinner 2016). Kade (1968, 56) lässt übrigens keinen Zweifel an der Motivation der Mitglieder der Leipziger Schule, die als subjektiv angesehene Kriterien nicht zu betrachten; dies darf aber nicht missverstanden werden als Überzeugung der Mitglieder der Leipziger Gruppe, diese Aspekte seien nicht von Relevanz!

Eine ganze Reihe von Aspekten, deren vermeintlich fehlende Berücksichtigung in den linguistischen Ansätzen allgemein und in der Leipziger Schule im Speziellen kritisiert worden ist, ist im Laufe der Jahre de facto sehr wohl in der Leipziger Schule behandelt und zum Teil auch ausführlicher betrachtet worden. Und dies geschah in einigen Fällen Jahrzehnte, bevor sie in anderen translationswissenschaftlichen Strömungen zur Sprache kamen, die heute gerade für ihre Berücksichtigung als besonders innovativ gelten. Die Kritik bezeugt somit weniger Mängel der Leipziger Ansätze als fehlende Einsicht in die Texte der Leipziger Schule und mangelndes Verständnis der Ansätze. Zu erwähnen sind unter anderem

- die Reflektion über die Übersetzungspraxis und ihre Bedeutung für die Theorie und umgekehrt die Relevanz der Theorie für das Übersetzen und somit die Berücksichtigung der Beziehung zwischen Übersetzer bzw. Übersetzerin und Übersetzungstheorie (s. hierzu bereits Neubert 1965, 83, s. auch Kade 1963 zu den Zielen der Translatologie);

- die theoretische Einbeziehung der auftragserteilenden Entität (welche oft als Innovation der funktionalistischen Übersetzungswissenschaft angesehen wird) und die Frage nach dem Handeln im Falle der Ablehnung der Aussage eines Textes durch die übersetzende Person (s. hierzu beispielsweise Wotjak 2002, 4 zu Kade 1980);

- das Verständnis der Übersetzung als Handlung, welches sich bereits bei Jäger (1975) findet, wird heute vor allem mit Holz-Mänttäri (1984) in Verbindung gebracht (s. ausführlicher Sinner 2016);

- die Frage der vermeintlich fehlenden Zieltextorientierung, die unserer Auffassung nach unbedingt gegeben war (s.u.).

Im Folgenden sollen zwei Aspekte genauer betrachtet werden, zu einem ein terminologischer Aspekt, der die offenbar mangelhafte Durchdringung der Leipziger seitens anderer Autoren und Autorinnen offenlegt, und eine die Ausrichtung der Leipziger Schule betreffende Fragestellung, an der das fehlende Verständnis oder die mangelhafte Kenntnis der Texte der Leipziger Schule sichtbar wird und zugleich ihre Innovationskraft herausgearbeitet werden kann.

\section{Translatologische Terminologiebildung als Beleg für die Theoriebildung: Sprachmittlung und Translation}

Eine Analyse translationswissenschaftlicher Arbeiten im Hinblick auf die Sicht auf die Terminologie der Leipziger Schule bzw. die Aussagen über Termini, die in der Leipziger Schule eine zentrale Rolle spielten, zeigt, dass die Originalquellen vielfach offenbar nicht eingesehen oder nur sehr oberflächlich konsultiert wurden und dass bestimmte Fehlein- 
schätzungen eher die Regel als die Ausnahme sind. Die von Autoren wie Jäger, Kade und Neubert eingeführte Terminologie spielte offenbar eine gewichtige Rolle in der unvollständigen bzw. der Negativrezeption der Postulate der Leipziger Schule. Auf zwei zentrale Termini soll daher im nachfolgenden umfassender eingegangen werden, darunter der Ausdruck zur Bezeichnung der Tätigkeit, von der sich letztlich der Name des Produktes der translatorischen Handlung und der Name der wissenschaftlichen Disziplin herleitete.

Die Verantwortlichen für die Leipziger Übersetzungstheorie prägten die wissenschaftliche Auseinandersetzung mit Übersetzen und Dolmetschen vielleicht in besonders sichtbarer Weise in terminologischer Form, wie das Verständnis der Studienobjekte und der damit konsequent einhergehende Gebrauch von Termini wie Translation, Translat oder Sprachmittlung bezeugt.

Anders als in modernen Übersichten über die Translatologie, wie etwa bei Prunč (2007) dargestellt, findet sich der Terminus Translation bereits in einem Aufsatz von Kade aus dem Jahr 1963; in Kade (1968) erfolgt dann eine systematische und explizite Definition der drei Termini Translation, Translat und Translator. Kade unterschied dabei zwischen Translation im engeren Sinne - das mündliche und schriftliche Übertragen eines Ausgangstextes in einen anderssprachigen Zieltext - und Translation im weiteren Sinne. Der in Westdeutschland zuerst lange Zeit als unnötiges Fremdwort abgelehnte Terminus erscheint in der Bedeutung, Übersetzung' schon im 15. Jahrhundert bei Heinrich Steinhöwel (1412-1483, unter anderem Übersetzer von Petrarca und Boccaccio), und wurde auch im Habsburgerreich verwendet (Prunč 2007, 13). In der wissenschaftlichen translatologischen Literatur ebenso wie in Arbeiten zur Sprachmittlung in der Didaktik finden sich bis heute zahlreiche Mängel hinsichtlich der Chronologie, der Herkunft und der genauen Definition bzw. der genauen Bedeutung der Termini, insbesondere von Sprachmittlung (s. die Darstellung einiger Fehleinschätzungen in Sinner - Wieland 2013).

Eine sehr übersichtliche und insbesondere sehr präzise Einordnung und Differenzierung des Terminus Sprachmittlung und eine klare Kontrastierung mit dem Terminus Translation finden sich in Jäger (1986), wo Translation explizit aus Sprachmittlung entwickelt wird. Jäger schreibt:

Die meisten Autoren allgemein-übersetzungswissenschaftlicher Darstellungen beschränken sich (gewöhnlich ohne diese Einschränkung zu vermerken) auf die kommunikativ äquivalente Sprachmittlung (vor allem auf das sog. adäquate Übersetzen) und entheben sich damit der Notwendigkeit, eine Bestimmung von Sprachmittlung zu geben, die auch auf die kommunikativ heterovalente Sprachmittlung zutreffen würde. [Es besteht] die Unklarheit darüber, [...] welche bzw. wieviele kommunikativ relevante Eigenschaften des quellensprachigen Textes im zielsprachigen Text erhalten bleiben müssen, damit dieser noch als Resultat eines Sprachmittlungsprozesses betrachtet werden kann, und wieviele kommunikativ relevante Eigenschaften ein entsprechender zielsprachiger Text haben darf, die dem quellensprachigen Text nicht zukommen, ohne daß er damit aufhört, Ergebnis sprachmittlerischen Handelns zu sein (Jäger 1986, 5-6).

Anschließend definiert Jäger Sprachmittlung dann ausführlicher:

[So] [...] kann man auch sagen, daß Sprachmittlung eine komplexe sprachliche Tätigkeit im Rahmen der sprachlichen Kommunikation ist, die zu dem Zweck vollzogen wird, die 
Kommunikation zwischen verschiedensprachigen Partnern zu gewährleisten, und darin besteht, daß zu einem LQ-Text (quellensprachigen Text) ein L2-Text (zielsprachiger Text) geschaffen wird, der sich hinsichtlich seiner kommunikativ relevanten Eigenschaften auf die kommunikativ relevanten Eigenschaften des LQ-Textes gründet, so daß der L2-Text den Kommunikationsbedürfnissen von Adressat(en) und/oder LQ-Text-Verfasser bezüglich kommunikativ relevanter Eigenschaften des LQ-Textes in dem Maße entspricht, wie dies unter den jeweiligen Bedingungen, unter denen die Sprachmittlung stattfindet, möglich ist. Bei dieser Fassung der Bestimmung von Sprachmittlung treten der zielsprachige und der quellensprachige Text und ihr gegenseitiges Verhältnis stärker in den Vordergrund (Jäger 1986, 6).

In den Erläuterungen zur Translation wird bei Jäger (1986, 9-10) dann auch die Beziehung zwischen Translation und Sprachmittlung deutlich:

Dafür sollen uns aber - im Sinne unseres Anliegens, die Translation als eine bestimmte Hauptart der Sprachmittlung näher zu bestimmen - einige Eigenschaften von Sprachmittlungsprozessen besonders interessieren, die nicht allen zukommen, sondern jeweils nur einer bestimmten Teilmenge, die aber insofern sehr bedeutsam sind, als sie

- spezifische Besonderheiten der jeweiligen Teilmenge darstellen, die in praktischer und/ oder theoretischer Hinsicht von speziellem Interesse sind und noch weitere Besonderheiten der jeweiligen Prozesse nach sich ziehen, und

- jeweils zu einer Gliederung der Gesamtheit aller Sprachmittlungsprozesse in zwei große Teilmengen führen und deshalb für eine Unterscheidung von Arten der Sprachmittlung sehr geeignet sind.

Auf Grund beider Charakteristika der zu betrachtenden Eigenschaften wollen wir die danach aufgestellten Arten der Sprachmittlung als Hauptarten verstehen. Mit der Annahme bestimmter Hauptarten der Sprachmittlung sehen wir zugleich eine bestimmte Klassifizierung der Sprachmittlungsprozesse vor. Wenn wir im weiteren vier Paare von Hauptarten:

- kommunikativ äquivalente und kommunikativ heterovalente Sprachmittlung,

- Übersetzen und Dolmetschen,

- Translation und Adaptation,

- Interpretation und Substitution,

annehmen und eine vierfache Bestimmung eines jeden Sprachmittlungsprozesses vorschlagen (so daß jeder Sprachmittlungsprozeß zu jeweils einer Hauptart von jedem Paar gehört), ist zu berücksichtigen, daß jede Klassifikation (verstanden als Ergebnis einer Klassifizierung) in dem Sinne relativ ist, daß das ihr entsprechende Erkenntnisinteresse und vor allem der ihr unterliegende Erkenntnisstand (bezüglich der für die Klassifizierung als relevant betrachteten Eigenschaften der Objekte) sich ständig entwickeln.

Es ist hier deutlich zu erkennen, dass Sprachmittlung in der Leipziger Schule bzw. der DDR allgemein weit über die enger gefasste Translation hinausgeht und somit beispielsweise nicht, wie oft fälschlich so dargestellt, als Synonym oder Überbegriff für Dolmetschen und Übersetzen verstanden werden darf (s. hierzu ausführlich Sinner - Wieland 2013). Wesentlich ist zudem, dass unter Sprachmittlung als Sonderform der Kommunikation, nämlich als zweisprachig vermittelte Kommunikation, von der Leipziger übersetzungswissenschaftlichen Schule, etwa Kade (1980), zwei klar zu differenzierende Phänomene verstanden wurden: einerseits die intralinguistische Sprachmittlung, in der 
ein Text $A$ in Sprache $L_{1}$ zu einem Text B in Sprache $L_{1}$ wird, und die interlinguistische Sprachmittlung, bei der aus einem Text $A$ in $\mathrm{L}_{1}$ ein Text $\mathrm{B}$ in $\mathrm{L}_{2}$ wird (s. ausführlich Jung 2000, 9-10). Und Kade (1980, 7) schreibt hierzu explizit:

Im Zuge der weiteren theoretischen Klärung der Vorgänge in der Translation, insbesondere bei der Untersuchung des Invarianzproblems und bei der Bestimmung des Gegenstandes der Übersetzungswissenschaft, wurde die Translation im Interesse einer genaueren Begriffsbestimmung als kommunikativ äquivalente Sprachmittlung charakterisiert. Ihr steht die kommunikativ heterovalente Sprachmittlung gegenüber, für die sich in der Praxis die Bezeichnung inhaltsbearbeitendes Übertragen eingebürgert hat.

Das Gegensatzpaar wird sehr deutlich, wenn Kade (1980, 75) schreibt:

Die Verwendung eines L1-Textes als Ausgangsgröße und die Erzeugung eines L2-Textes als Endgröße gehören deshalb zu den wesenhaften bzw. wesensbestimmenden Merkmalen der Sprachmittlung. Dies gilt sowohl für die Translation als auch für die heterovalente Sprachmittlung (das ,inhaltsbearbeitende Übertragen') [...] (meine Hervorhebung).

Die seit den 2000er Jahren in der Fremdsprachendidaktik aufgekommene Sprachmittlung ist, so wie sie heute aufgefasst wird, nur bedingt im Sinne der Leipziger Schule zu verstehen (offenbar rezipiert auch die moderne Fremdsprachendidaktik die Leipziger Schule nicht oder nur mangelhaft), sondern beinhaltet einerseits die Ebene des nicht professionellen Sprachmittelns (vgl. hierzu etwa Knapp - Knapp-Potthoff 1985) und andererseits ganz offensichtlich auch das in dem von Kade und den anderen Vertretern der Leipziger Schule vertretenen Ansatz der Sprachmittlung als heterovalente Sprachmittlung bezeichnete inhaltsbearbeitende Übertragen (Sinner - Wieland 2013).

Das Beispiel der Terminologie für wesentliche Aspekte der Klassifizierung der Gegenstände und der Reichweite der Untersuchung zeigt deutlich den wesentlichen und bis heute vergleichsweise erstaunlich modern und umfassend erscheinenden Beitrag zur übersetzungswissenschaftlichen Theorienbildung und zugleich, dass die Leipziger Übersetzungswissenschaftler und Übersetzungswissenschaftlerinnen ihren Untersuchungsbereich weit über die rein linguistische Betrachtungsweise hinaus erfassten.

\section{5. (Fehlende) Zieltextorientierung als Stein des Anstoßes}

Eine fast schon stereotype Kritik an der Leipziger Schule betrifft ihre Sicht auf die Rolle von Zieltext und Ausgangstext. Angeblich wurde die Ausrichtung der translatologischen Betrachtung auf den Zieltext nach und nach eingeführt bzw. erweitert; während die linguistischen Ansätze in einer Fixierung auf den Ausgangstext verharrten, sei auf Initiative der funktionalistischen und einiger der deskriptiven Ansätze und im Anschluss an die pragmatische und kulturelle Wende der Zieltext immer mehr ins Zentrum der Aufmerksamkeit gerückt (s. Sinner 2016). Tatsächlich aber liegt auf der Hand, dass mit der außerordentlich frühen Berücksichtigung pragmatischer Aspekte durch die Leipziger Schule ein eigentlich wohl ausreichender Beweis für ihre diesbezügliche Vorreiterrolle in 
der Translatologie erbracht ist und dass die Zieltextorientierung dem Leipziger Ansatz keineswegs fremd war.

Nicht nur geht die Frage der grundsätzlichen Übersetzbarkeit aus Sicht der Leipziger Schule unzweifelhaft über das Linguistische hinaus. So konstatierte Neubert (1986, 89) schon in den Anfängen der übersetzungswissenschaftlichen Betätigung in Leipzig: „Einem möglichen Mißverständnis muß jedoch zum Schluß noch vorgebeugt werden: Die Übersetzbarkeit ist nicht nur ein linguistisches, sondern auch ein pragmatisches Problem".

Neubert ist zudem der erste, der - schon 1968! - einen wissenschaftlichen Beitrag zu pragmatischen Aspekten der Übersetzung veröffentlicht, also schon Jahre vor dem Aufkommen explizit zieltextorientierter Ansätze an das Übersetzen (vgl. Bernardo 2009, 334-339 zu den pragmatischen Tendenzen der Leipziger Schule). Auch Kade erklärt, dass der Adressat ein wesentliches Kriterium für die Texterstellung darstellt:

Schließlich wirken in der Kommunikation und somit auch in der Translation Faktoren, die aus der Relation zwischen Zeichen und Zeichenbenutzer resultieren: Mit jeder sprachlichen Äußerung verfolgt ihr Verfasser einen bestimmten Zweck, der die Auswahl der Mittel des Kodes beeinflußt. Jede Mitteilung ist an einen bestimmten Adressaten gerichtet, was ebenfalls die Form der Mitteilung bestimmen kann. Sprachliche Äußerungen kommen nie isoliert von einer bestimmten Situation und einem bestimmten, historisch gewachsenen sozialen und kulturellen Hintergrund vor. Situationskontext oder historischer Kontext können dafür entscheidend sein, welche sprachlichen Mittel zur Realisierung eines bestimmten Inhalts in einem konkreten Kommunikationsakt aufgewendet werden. Aus der Zeichen-Zeichenbenutzer-Relation ergeben sich die pragmatischen Aspekte der Translation (Kade 1968, 51).

Noch früher hebt Neubert (1965) im Zusammenhang mit seinen Regeln des Übersetzens - wohl paraphrasierbar als Wege zur Erlangung einer bestimmten Übersetzung hervor, dass die Vorgehensweise je nach dem Zweck einer Übersetzung anders sein kann, was zu einer Modifizierung der Regeln führen könnte („Bei Beachtung des Zweckes einer Übersetzung müssen die angeführten 8 Regeln sicherlich noch modifiziert werden“; Neubert 1965, 89). Und mit der Vorgehensweise kann sich natürlich auch die Haltung zur Art und Weise ändern, wie eine Aussage in der Zieltextkultur am besten auszudrücken ist, um ein bestimmtes Ziel zu erreichen - und das entspräche der Idee des Skopos, mit der später die Funktionalisten auftrumpften.

Auch Gerd Wotjak äußert sich diesbezüglich, wenn er schreibt, „das Konzept der kommunikativen Äquivalenz [beinhalte] durchaus viele mit konkurrierenden, zeitlich frühestens in den 80er Jahren liegenden und gegenwärtig häufig dominierenden translatologischen Beschreibungsansätzen kompatible Aspekte [...]“ (Wotjak 2002, 4; s. auch Wotjak 2002-2003).

Die Reflektionen der Vertreter der Leipziger Schule zur kommunikativen Äquivalenz werden heute immerhin auch von einigen Autoren und Autorinnen, die ansonsten die starke Ausrichtung auf linguistische Aspekte als Defizit sehen, als maßgeblich oder entscheidend angesehen (s. etwa Bolaños Cuéllar 2008, 5). So kann es nicht verwundern, dass Wotjak $(2002,12)$ es für notwendig erachtet, die mangelhaften Interpretationen der kommunikativen Äquivalenz zu überwinden, die in der Translatologie aufgrund einer 
insgesamt unzureichenden Rezeption dieses der Leipziger Schule zugrunde liegenden Gedankens in übersetzungswissenschaftlichen Abhandlungen (wie etwa der besonders unangemessenen Darstellung bei Stolze 1994) noch immer bestehen.

Wie Heide Schmidt (1987) darlegt, muss ein übersetzter Text ebenso wie auch ein Text, der aus einer heterovalenten Bearbeitung bzw. Adaptation (und das war für die Leipziger Schule keine Übersetzung) hervorgeht, kommunikativ angemessen sein. Das heißt, sie müssen die Anforderungen an die Textkonventionen erfüllen, die im jeweiligen Fall für die Erstellung der entsprechenden Textsorte gelten. Eine optimale Übersetzung müsste kommunikativ äquivalent zum Ausgangstext und kommunikativ angemessen im Hinblick auf die Textkonventionen und Traditionen der Texterstellung in der Zielsprache sein (s. Wotjak 2002, 12).

Wenngleich die Leipziger Schule wie gesehen außersprachliche und subjektive Faktoren zwar erkannte, sie aber explizit nicht als Objekt ihrer Untersuchungen ansah, so finden wir doch bei ihren Vertretern und Vertreterinnen ein ausgeprägtes Bewusstsein für die verschiedenen außersprachlichen und ,individuellen“ Aspekte der Textproduktion und der Textrezeption (bzw. Textperzeption).

Die individuelle Textproduktion setzt die grundsätzliche Fähigkeit der einzelnen Person voraus, einen Text so zu verfassen, dass er von anderen verstanden werden kann, denn das ist das vorgeordnete Ziel jeglicher Kommunikation. Umgekehrt setzt die individuelle Textrezeption voraus, dass die einzelne Person in der Lage ist zu entscheiden, welches Verständnis des vorliegenden Textes möglich oder wahrscheinlich ist, also auch zu entschlüsseln, mit welcher kommunikativen Absicht der Text wahrscheinlich verfasst wurde. Die Idee von der individuellen Fähigkeit zur Textproduktion schließt jedoch nicht aus, dass der Text individuelle Charakteristika aufweist, die mit der Person zusammenhängen, welche den Text verfasst hat, oder Eigenschaften hat, die auf die soziale Gruppe, der sie angehört, verweisen, also beispielsweise diatopisch oder diastratisch markierte Elemente.

Jede Betrachtung aus variationsorientierter linguistischer Perspektive muss Oksaar (2000) zufolge die Analyse des Idiolektes (als individuelle Realisierung des sprachlichen Systems) umfassen, da alle Varietäten stets idiolektal beeinflusst sind (vgl. Oksaar 1987). Da Sprache und ihre Varietäten immer durch individuelle Realisierungen entstehen, muss gemäß Oksaar diese individuelle Umsetzung der grundlegende Ausgangspunkt für die Bestimmung auch des kollektiven Verhaltens sein. Idiolekte haben aber, und das ist nicht weniger relevant, auch eine besondere Bedeutung für die Wahrnehmung sprachlicher Äußerungen. Noch immer gilt Dittmars (1997, 182-183) Einschätzung, wonach Idiolekte in dieser Hinsicht so gut wie nicht analysiert worden sind (s. Sinner 2014, 149), und dies muss unbedingt auch für die Translatologie gelten (s. Sinner - Morales 2015).

Tatsächlich war jedoch bereits für die Leipziger Schule der Einfluss der Charakteristika des Individuums und insbesondere der idiolektalen Unterschiede zwischen Sender und Empfänger ein wichtiger zu berücksichtigender Aspekt (wenngleich er nicht den Kern ihrer Analyseinteressen berührte), denn ihre Vertreter und Vertreterinnen verstanden Translation als einen Prozess der zweisprachigen, durch eine dolmetschende oder übersetzende Person vermittelten Kommunikation (s. o.). Kade $(1968,52)$ schreibt, unter Verweis auf Ammer (1961): 6

6 Ammer, Karl: Sprache, Mensch und Gesellschaft, Halle (Saale): Sprache und Literatur 1961, s. 59. 
Abgesehen von solchen Abweichungen des Kodes, die daraus resultieren, daß die Kode-Systeme, aus denen S und E ihre Mittel schöpfen, gewisse Unterschiede aufweisen, besteht im konkreten Kommunikationsakt zwischen dem Kode von S und dem Kode von E niemals völlige Übereinstimmung, auch wenn sie ihre Kommunikationsmittel ein und demselben System entnehmen, weil der Idiolekt von S gegenüber dem Idiolekt von E in jedem Falle Abweichungen aufweist.

Zweifellos ist mit der Frage nach dem Verständnis auf Zieltextseite neben der Betrachtung der Ausgangstextseite unbedingt auch eine Zieltextorientierung gegeben, wenngleich diese, im Rahmen der Theoriebildung der Leipziger Schule selbstverständlich, nie stärker als die Ausgangstextorientierung war.

Die Frage nach der Perzeption ist außerordentlich zukunftsweisend und kann als Forderung nach perzeptiver Translatologie avant la lettre angesehen werden.

\section{Perzeptive Translatologie. Eine exemplarische Betrachtung anhand von Jäger (1975)}

\subsection{Perzeptive Translatologie}

In den letzten Jahren ist mit der Zuwendung zur perzeptiven Analyse von Ausgangsund Zieltexten unter Einbezug von Analysen der Perzeption von muttersprachlichen Texten in Ausgangs- und Zielsprache durch jeweils muttersprachliche Testpersonen eine Ausrichtung der Translatologie vorgenommen worden, die in den frühen Perspektiven der Leipziger Schule überraschend „moderne“ Anknüpfungspunkte findet. Die perzeptive Translatologie (Sinner - Morales 2015, Sinner 2019) geht von der Prämisse aus, dass Perzeptionsanalysen auf Textgrundlage und mit jeweils muttersprachlichen Testpersonen beider Sprachen erforderlich sind, wenn festgestellt werden soll, ob ein übersetzter Text tatsächlich dieselbe Wirkung hat wie der Ausgangstext bzw. ob in dem (in der Praxis wohl eher selteneren) Fall, dass der Skopos eine Änderung des Zweckes bzw. der Wirkung verlangt, eben diese intendierte Änderung der Wirkung erzielt wurde (zu den Zielen und Möglichkeiten der perzeptiven Translatologie s. ausführlich Sinner - Morales 2015 und Sinner 2019). In der Dolmetschwissenschaft wird vereinzelt bereits nach der content validity, also dem quantifizierbaren Anteil des durch die Verdolmetschung erfassten Inhalts, gefragt. Die im Qualitätsassessment in anderen Lebensbereichen, etwa der Werbung, schon länger üblichen Wirkungsanalysen sind aus translationswissenschaftlicher Perspektive noch immer als Desiderat anzusehen.

Genau solche Wirkungsanalysen forderten nun die frühen Vertreter der Leipziger Übersetzungswissenschaftlichen Schule, wenn sie von einer Berücksichtigung der Rezeption sprachen oder nach der Rolle der Empfänger bzw. der Empfängerinnen und nach dem Verhältnis zwischen den sprachlichen Gegebenheiten einerseits und den Nutzern und Nutzerinnen auf Empfängerseite sowie der Gerichtetheit des Textes andererseits fragten (Neubert 1968). Tatsächlich hat diese Herangehensweise sehr viel gemein mit dem, was man in später als funktionalistisch klassifizierten Arbeiten unter dem Namen Skopos als vermeintliche Neuerung in die Übersetzungswissenschaft einbrachte (s. Jung - Sinner -Batista 2013, 9). 
Zusätzlich zu (oder vielleicht sogar anstelle von) einem Vergleich von Ausgangs- und Zieltext ist der Vergleich der Wirkung des Ausgangstextes mit der Wirkung der Übersetzung (oder mehrerer möglicher Übersetzungen) für eine Bewertung einer Übersetzung hinsichtlich ihrer "Gelungenheit" eigentlich unabdingbar. Das Problem ist, dass die Einschätzung der Wirkung in erster Linie ein Sprecherurteil ist. Genau wie die Einschätzung von nicht „objektiv“ feststellbaren, also nicht „eindeutigen“ Fehlern hängt auch die Bestimmung der Wirkung vom Urteil der bewertenden Personen, also von einem Sprecherurteil, ab.

Zur Bestimmung oder Beurteilung der Qualität von Übersetzungen vertraut die Kritik noch immer vor allem auf formale Aspekte und - als Erbe der generativen Ansätze - auf das eigene Urteil. Das ist besonders bemerkenswert bzw. problematisch, wenn man berücksichtigt, dass bereits die Korrektur von Übersetzungen durch den Übersetzer bzw. die Übersetzerin selbst als für die Qualitätssicherung nicht ausreichende Maßnahme angesehen wird (zur Frage des Qualitätslektorats s. Didaoui 2006).

Vor allem außerhalb generativ bzw. formalisierend arbeitenden Arbeitsbereichen der Sprachwissenschaft ist man sich längst einig darüber, dass einzelne Sprecherurteile nur einen sehr eingeschränkten Blick auf sprachliche Phänomene erlauben und im Hinblick auf varietätenlinguistische Aspekte zu überprüfen und somit auch um andere Perspektiven zu ergänzen sind. Dennoch vertrauen nicht wenige in vielen Bereichen bzw. in vielen Studien - und das gilt auch für die Translatologie - noch immer beharrlich auf den Wert einzelner Sprecherurteile bzw. auf Introspektion, also die Berücksichtigung nur des eigenen Urteils. Bis heute ist der unbedingte Verlass auf die eigene Meinung und der damit (natürlich) verbundene Mangel an Empirie einer der Hauptkritikpunkte an Chomsky und den ihm nachfolgenden Linguisten und Linguistinnen.

Obwohl von Vertretern der Leipziger Schule wie gesehen schon seit den 1960er Jahren auf die Bedeutung der Wirkung von Ausgangs- und Zieltext verwiesen wurde, ist die Rezeptions- bzw. Perzeptionsanalyse in translatologischen Studien noch immer die Ausnahme (s. Sinner 2014, Sinner - Morales 2015). Zwar haben sich neben den Vertretern und Vertreterinnen der Leipziger Übersetzungswissenschaft auch andere Autoren und Autorinnen mit der Frage der Wirkung auseinandergesetzt, das hat aber nicht zu empirischen Analysen von beabsichtigter und tatsächlich erzielter Wirkung geführt. Wie Wotjak $(2002,13)$ ausführt, ist hervorzuheben, dass die Leipziger Schule nicht nur die Produktion von Texten, sondern auch ihre Rezeption bzw. die Art und Weise, wie sie verstanden oder dekodiert bzw. „entschlüsselt“ werden (Kade 1968), als wichtig erachtet. Wotjak ergänzt, dass die Leipziger Schule neben der Perspektive des Rezipienten/ Hörers bzw. der Rezipientinnen/Hörerinnen und dem Übergang vom Interesse an der Bedeutung des Ausgangstextes zur Produktion eines kommunikativ äquivalenten Textes (was offensichtlich auf einer onomasiologischen Sicht beruht) neben den kommunikativen Aspekten auch metakognitive Fragestellungen thematisiert. Und eben dieser letzte Punkt, so der Autor, sei in den bis in die 1980er Jahre durchgeführten dolmetschwissenschaftlichen Untersuchungen der Leipziger Schule noch deutlicher zu spüren.

Die Vorteile von Perzeptionsstudien beispielsweise für die Annäherung an Qualitätskriterien in der Übersetzungswissenschaft liegen auf der Hand. Insbesondere der Umstand, dass durch die Befragung von muttersprachlichen Testpersonen individuelle und, darauf aufbauend, gruppenspezifische Assoziationen und Interpretationen identi- 
fiziert und berücksichtigt werden können, ist für eine über individuelle Urteile hinausgehende Erschließung von Qualitätsmerkmalen von Übersetzungen relevant. Von Relevanz sind auch die Möglichkeiten der Inbezugsetzung der potentiellen Effekte von Übersetzungen mit ihrem Erfolg in der jeweiligen Zielkultur.

Genau wie es die linguistischen Ansätze in den frühen Tagen der Übersetzungswissenschaft ermöglichten, durch sprachpaarbezogenen Sprachvergleich und Übersetzungsanalysen Regelmäßigkeiten und Besonderheiten in bestimmten Übersetzungspaaren zu ermitteln, oder wie es die Korpuslinguistik erlaubte, die textlichen Charakteristika von Ausgangs- und Zieltexten zu beschreiben und zu bewerten und Universalien oder Übersetzungshaftigkeit von Texten zu ermitteln (s. Sinner 2014, 255-256), so können vergleichende Perzeptionsstudien von Ausgangs- und Zieltext einen wichtigen Beitrag zur Ermittlung grundlegender Tendenzen des Verstehens von Äußerungen im Kontext leisten und kann die perzeptive Translatologie durch Wirkungs- und Verständnisanalysen zur Ermittlung von Regelmäßigkeiten und Auffälligkeiten im Hinblick auf die Veränderung oder Verschiebungen von Wirkung im Kontrast von Original und Übersetzung herangezogen werden.

Es liegt auf der Hand, dass Perzeptionsstudien, und insbesondere Perzeptionsstudien größeren Umfangs oder gar repräsentativer Art, in der alltäglichen Übersetzungspraxis nicht durchführbar sind, aber dieser Ansatz kann ebenso wie die genannten vergleichenden Studien bestimmter Sprachpaare dazu beitragen, Regelmäßigkeiten im Übersetzungsprozess zwischen bestimmten Sprachen und in der Wirkung bestimmter sprachlicher Strukturen auf unterschiedliche Gruppen zu ermitteln und so auch Rückschlüsse für die Ausbildung im Bereich der Translation zu ziehen.

\subsection{Der Abzug der Truppen oder: Perzeptive Translatologie avant la lettre}

Ein besonders aussagekräftiges Beispiel für die Relevanz der Frage nach der Bedeutung bzw. nach dem Verständnis eines deutschen Ausgangstextes im direkten Vergleich mit der Wirkung von mehreren tschechischen Zieltextversionen bei Jäger (1975) soll zur Illustration der im Hinblick auf die Berücksichtigung des Verständnisses zukunftsweisenden Positionen der Leipziger Schule nachfolgend genauer betrachtet werden.

Jägers Text von 1975 wird zusammen mit Texten wie beispielsweise den Arbeiten von Neubert (1965, 1986), Kade (1968) oder Schmidt (1987) als besonders bedeutend für die Herausbildung der Leipziger Schule angesehen und kann als Teil eines ähnlich gelagerten Diskurses, als Schlüssel- oder Referenztext für die gesamte durch einen gemeinsamen Erinnerungshorizont geprägte Textserie gelten (Sinner 2016, 134-135). ${ }^{7}$

7 Diskurs ist hier im Sinne eines virtuellen Textkorpus zu verstehen, das durch den Inhalt und den Forschungsgegenstand der Texte bestimmt wird, die eindeutig aufeinander bezogen sind (Busse Teubert 1994, 14). Durch eine Analyse der Texte können Anzeichen für die bewusste oder unbewusste Orientierung an anderen Autoren und zeitlich vorhergehenden Diskurs- oder Vertextungstraditionen im Sinne des horizon de rétrospection der Autoren herausgearbeitet werden; dieser „Erinnerungshorizont“ ist im Sinne Auroux' $(1996,31)$ als Gesamtheit der Texte und Autoren zu verstehen, die bei der Bearbeitung eines Themas über die Erinnerung aktualisiert werden. Unter Textserie wird nach Haßler $(2000,2002)$ eine Gesamtheit von individuellen Texten verstanden, die sich mit der gleichen Materie im gleichen erkenntnistheoretischen Zweig mit nicht unbedingt gleicher Methodik, aber mit 
Jäger untersucht verschiedene Gründe dafür, dass es mehrere Translate zu einem Original geben kann, und formuliert die grundsätzliche Frage, „ob es zu einem $\mathrm{L}_{\mathrm{Q}}$-Text [= Ausgangstext $]$ nur einen kommunikativ äquivalenten $\mathrm{L}_{\mathrm{Z}}$-Text [= Zieltext] gibt ${ }^{\text {" }}$ $(1975,56)$ und, da die Antwort negativ ausfällt, „unter welchen Bedingungen unterschiedliche Texte kommunikativ äquivalent sein können“ (1975, 56). In diesem Zusammenhang betrachtet er auch den Umstand, dass unterschiedliche Übersetzungen im Tschechischen dieselbe Äquivalenz mit dem deutschen Original aufweisen können, obwohl sie untereinander eindeutig unterschiedlich sind, und befasst sich somit mit dem Problem, inwiefern damit Synonymie bzw. Polysemie ein Problem für die kommunikative Äquivalenz darstellen kann. Die für Sprachen charakteristische Existenz partieller Synonyme und die damit verbundene Vagheit der sprachlichen Zeichen stellt dabei eine erste Ursache dar. Gehe man davon aus, dass Synonymie im lexikalischen und syntaktischen Bereich im Wesentlichen funktionalstilistisch sei, so müssten sich zwei Zieltexte, die sich nur darin unterscheiden, dass sie neben identischen Elementen auch solche umfassen, die in einer Synonymiebeziehung zueinander stehen, in ihrem kommunikativen Wert unterscheiden, da sich die Zugehörigkeit zu einem bestimmten Funktionalstil im kommunikativen Wert reflektiere $(1975,57)$. Er entkräftet die Annahme, indem er anhand eines deutsch-tschechischen Beispieles zur Illustration der Existenz von kommunikativ äquivalenten Transpositionen und somit von mehr als einem Translat aufzeigt, dass beispielsweise syntaktische Synonyme nicht in jedem Falle funktionalstilistisch differenziert seien:

In der Erklärung der ZK der Parteien und der Regierungen der Staaten der sozialistischen Staatengemeinschaft zur Lage im Nahen Osten vom November 1969 hieß es z.B.: "Die Völker der Welt müssen Israel zwingen, seine Truppen ... abzuziehen" und "... die Resolution ..., die Israel verpflichtet, seine Truppen ... abzuziehen ..." (Neues Deutschland, 27. Januar 1969). Die tschechische Version lautet einmal "Národy světa musí přimět Izrael, aby stáhl svá vojska ...", einmal "... rezoluci, která zavazuje Izrael ke stažení vojsk..." (Rudé právo, 27. November 1969). Die prädikativische und die nichtprädikativische Konstruktion sind in beiden Fällen austauschbar, so daß sich auch hier, wenn wir das Deutsche als Original annehmen, nicht nur ein tschechisches Translat ergeben würde. (Jäger 1975, 59)

Nach einem spanisch-deutschen Beispiel für die Existenz nicht nur eines Translates aufgrund der Synonymie in der Zielsprache, die aufgrund der Vagheit zweier in Bezug auf das ausgangssprachliche Zeichen tarde - por la tarde, a eso de las seis - komplementärer zielsprachlicher Zeichen - am späten Nachmittag, etwa gegen sechs Uhr oder am frühen Abend, etwa gegen sechs Uhr - entstehe (Jäger 1975, 59), führt Jäger wieder ein deutsch-tschechisches Beispiel an und bestimmt die Mehrdeutigkeit des deutschen Ausdrucks Abzug aufgrund der unterschiedlichen tschechischen Übersetzung: Abzug interpretiert nach dem transitiven Verb etwas/jmd. abziehen vs. Abzug interpretiert nach dem intransitiven Verb abziehen. Er formuliert dies so:

vergleichbarem Zweck und unter vergleichbaren Bedingungen auseinandersetzen (s. insbesondere Haßler 2002, 561-562). 
Eine zweite Quelle für die Existenz mehrerer Translate zu einem Original [neben dem Fall, dass aus der Vagheit zweier (im Hinblick auf das quellensprachliche Zeichen) komplementärer zielsprachlicher Zeichen eine Synonymiebeziehung zwischen ihnen entsteht] bedeuten die nichtmonosemierbaren Polysemien in den Originalen. Betrachten wir dazu zunächst ein Beispiel. In einem Satz wie "Die Völker müssen Israel zum Abzug seiner Truppen zwingen" oder "Der Einmarsch und der Abzug der Truppen geschah in den Nachtstunden" ist die der Wortgruppe "Abzug der Truppen" zugrunde liegende Tiefenstruktur ermittelbar, die es gestattet festzustellen, daß beim ersten Satz 'Abzug' mit dem transitiven Verb 'etwas/jmd. abziehen', beim zweiten Satz mit dem intransitiven Verb 'abziehen' in Verbindung gebracht werden muß. (Jäger 1975, 59-60)

Im Kontext wird nicht deutlich, ob es sich bei der Losung „Die Völker müssen Israel zum Abzug seiner Truppen zwingen“ um ein lediglich nicht verortetes Zitat aus einer deutschen Tageszeitung handelt oder ob Jäger erneut, aber in reformulierter Form aus der zuvor schon zitierten Erklärung der Zentralkomitees der Parteien und der Regierungen der Staaten der sozialistischen Staatengemeinschaft zur Lage im Nahen Osten vom November 1969 zitiert; das zweite Beispiel „Der Einmarsch und der Abzug der Truppen geschah in den Nachtstunden" dagegen ist offenbar konstruiert. In jedem Fall aber ist das Beispiel mit Israel sicherlich im Licht der damaligen politischen Sicht der sozialistischen Länder gegenüber Israel (vgl. Lerman 2019) und somit auch in der Art der Berichterstattung in den DDR-Medien zu sehen. Jäger schreibt weiter:

Werden diese Sätze ins Tschechische transferiert, entspricht 'Abzug' im ersten Satz 'stažení', im zweiten Satz 'odchod'. Jeder der beiden Sätze ist nur auf eine Weise zu transferieren. Betrachten wir aber den Satz "Der Friedensvorschlag der Regierung der R[epublik]S[üd] V[ietnam] enthält als ersten Punkt den vollständigen Abzug der Truppen der USA und ihrer Verbündeten aus Südvietnam und den anderen Gebieten Indochinas", so ist 'Abzug' polysem. Da aber im Tschechischen kein auf dieselbe Weise wie ,Abzug' polysemes Zeichen existiert und andererseits auch ein Bezug auf die Wirklichkeit zu keinem anderen Ergebnis führt, als daß beide Bedeutungen nicht nur sprachlich, sondern auch sachlich möglich sind, müssen wir sowohl ein Translat mit ,stažení als auch ein Translat mit ,odchod' (beide Zeichen werden in diesem Zusammenhang auch häufig in der Presse verwendet) akzeptieren. Allerdings führt uns dieser Fall zu der Frage, ob es sich hier noch um eine kommunikative Äquivalenz handelt. (Jäger 1975, 59-60)

Obwohl Jäger die Bedeutung der Bedeutung und Wirkung der verschiedenen Elemente eines Textes bewusst war, geht auch er den Möglichkeiten der Ermittlung dieser Wirkung nicht auf den Grund. Eine Perzeptionsstudie zur Ermittlung der Frage, wie andere Personen mit Deutsch als Muttersprache die beiden Sätze verstehen, zieht er nicht in Betracht. Dies muss wohl mit der zu dieser Zeit vorherrschenden Sicht in Zusammenhang stehen, wonach eine muttersprachliche Meinung - also hier die der forschenden Person - ausreicht.

Auf Grundlage der beiden hier von Jäger kontrastierten Sätze führten wir daher von März bis Juni 2020 eine Befragung von 24 Muttersprachlern und Muttersprachlerinnen des Deutschen zum Verständnis von Abzug in einigen der Beispiele Jägers und in weiteren Äußerungen durch. Alle Testpersonen können aufgrund ihrer Ausbildung oder Arbeit als für Sprache überdurchschnittlich sensibilisiert angesehen werden, als Multi- 
plikatoren sprachlicher Gewohnheiten und als Angehörige der sprachlich und kulturell hegemonialen Schicht der Gesellschaft (vgl. Guespin 1985 und Bochmann 1993, 20) gelten (s. zu diesen Kriterien ausführlich Sinner 2004, 186-197). Die Testpersonen waren zwischen 24 und 77 Jahre alt und in jeder der sechs Altersgruppen (21-30, 31-40, 41-50, 51-60, 61-70 und 71-80) wurden jeweils zwei Männer und zwei Frauen befragt, so dass insgesamt zu gleichen Teilen Männer und Frauen aller berücksichtigten Altersgruppen berücksichtigt wurden. Das Verständnis wurde ermittelt, indem in einem ersten Schritt um eine Umformulierung der Strukturen zur Erklärung ihrer jeweiligen Aussage gebeten und in einem zweiten Schritt nach den Unterschieden zwischen den Strukturen gefragt wurde:

i. Die Völker müssen Israel zum Abzug seiner Truppen zwingen

ii. Der Einmarsch und der Abzug der Truppen geschah in den Nachtstunden

iii. Der Friedensvorschlag [...] enthält als ersten Punkt den vollständigen Abzug der Truppen der USA und ihrer Verbündeten aus [...] [diesen] Gebieten [...] (Jäger 1975, 59).

Nach diesen drei Sätzen wurden den Testpersonen anschließend zwei weitere (s.u.) vorgelesen bzw. vorgelegt.

Abzug ist zwar, wie Jäger in seinen Ausführungen ja zeigt, zweifellos polysem, wurde aber von den Informanten und Informantinnen der Studie immer und in der anschließenden Auswertung auch klar ersichtlich als Substantiv zu einem transitiven Verb assoziiert bzw. interpretiert.

Die Ergebnisse sind eindeutig: Durchweg alle Informanten und Informantinnen formulierten im Laufe der Interviews auf eine oder andere Weise, dass es bei Abzug um einen Befehl geht, die derzeitig besetzten Stellungen zu verlassen und sich, wohin auch immer, zurückzuziehen. Keine einzige Testperson assoziierte oder erläuterte Abzug allein mit dem intransitiven Verb abziehen. Nur drei Testpersonen erwähnten in einer Reformulierung oder einer Erklärung überhaupt beide Varianten, intransitives abziehen und transitives jmd./etwas abziehen, erklärten aber auf eine oder andere Weise, dass bei einem Heer bzw. einer Armee vorauszusetzen ist, dass die Stellung nur dann verlassen wird, wenn es einen entsprechenden Befehl gibt. Auf Nachfrage wurde von einer der drei Testpersonen sogar noch präzisiert, dass einzelne Soldaten beschließen könnten, ohne einen Befehl zu gehen, also zu desertieren, aber dass das eben nicht abziehen sei.

Einige Auszüge sollen den gewonnenen Gesamteindruck illustrieren.

Stefan, 50, Deutschlehrer

Damit ist gemeint, dass die anderen Länder Israel zwingen sollen, die eigenen Truppen von da, wo sie sind, zurückzuziehen. Also die Regierung von Israel. Die Länder sind hier die Handelnden, die Israel dazu bringen sollen, die Armee von da, wo sie ist, wegzubringen. Also wenn die so lange die Truppen bombardieren, bis sie abziehen, dann wäre das auch, „zum Abzug zwingen“, aber dann zwinge ich die Truppen zum Abzug, nicht Israel. Dass heißt, ich zwinge sie zu gehen. Israel kann man vielleicht durch Wirtschaftssanktionen dazu bringen, die Truppen abzuziehen, aber ich kann nicht Israel zwingen, dass die Truppen von alleine abziehen. Also, wenn ich Israel zum Abzug zwinge, dann befiehlt die Regierung, dass die Truppen abziehen. Es sind mehrere Akteure. 
Helga, 77, pensionierte Deutschlehrerin

Wie versteh ich das? Da ist ein Abzug, also dass die Truppen abgezogen werden. Das ist das, was Trump gerade in Deutschland macht, er zieht die Truppen ab und schickt sie nach Belgien.

Lilli, 28, Texterin

Die US-Truppen müssen aus diesen Gebieten herausgeholt werden. Also, sie müssen diese Gebiete verlassen, das muss die Regierung befehlen. Oder die Heerleitung. Heeresleitung. Heeresführung? Also die Generäle. Die müssen das befehlen. Und denen befiehlt es die Regierung. Jetzt wäre das Trump, der befiehlt. Per Twitter $<$ lacht $>$ und dann ziehen die Truppen ab.

Die Erklärungen lassen in der Regel leicht erkennen, welche Bedeutung von Abzug die Testpersonen verstanden haben bzw. zu definieren suchen. Auch in den folgenden Auszügen aus Aussagen zu allen drei Beispielen ist dies gut nachvollziehbar:

Katja, 50, Spanischlehrerin

Das bedeutet, dass Israel .../ die Völker, die Welt, Israel zwingen müssen, die Truppen da, wo sie sich niedergelassen haben, ab[zu]ziehen. Die Truppen sollen da weg. So hab ich es verstanden, beim Nur-Hören. Abzug ist für mich: weg, raus... von etwas wegziehen, wegholen wo sie jetzt sind, entfernen von etwas. Da soll/ die Völker sollen eine Entscheidung treffen, dass das passiert, das verstehe ich. Eine Aufforderung an die Völker ist das, also Appellcharakter.

$[\ldots]$

Der zweite Satz irritiert mich. Das heißt für mich/ ich sag mal zwei Ideen: Dass das alles an einem Tag, in einer Nacht passierte, sie sind sowohl hin als auch weg, wurden also in derselben Nacht gleich wieder abgezogen. Oder hab ich was falsch verstanden? Es könnte an einem Tag passieren oder irgendwann, nachts. Also sie kamen tags und wurden nachts dann abgezogen?

[...] Im dritten Satz sehe ich dasselbe, wenn die USA ihre Truppen nicht abziehen, wird es keinen Vertrag geben. Oder nein, falsch. Wenn die Truppen nicht rausgeholt werden, brechen sie den Vertrag. Also die USA.

Hier wird besonders gut deutlich, dass die Meinung Jägers, wonach der dritte Satz beide Perspektiven enthält und eindeutig polysem ist, durch die Aussage der Informantin nicht gestützt wird. Derselbe Eindruck entsteht in den folgenden Beispielen für Antworten zu Satz iii:

Myriam, 34, Korrekturleserin

Gemäß dem Entwurf für das Abkommen sollen die Vereinten Staaten ihre Truppen/ ihre Soldaten da/ also aus Vietnam herausholen.

Helga, 77, pensionierte Deutschlehrerin

Die USA müssen also die Truppen abziehen. Das ist wieder wie vorher [in Satz i], wie Trump, der die US-Truppen aus Deutschland abzieht.

Für die Testpersonen scheint klar zu sein, dass ein Abzug einen Befehl voraussetzt, und in einigen Fällen wird dies auch ausführlicher dargelegt, etwa im folgenden Beispiel, wo der Informant verschiedenen mögliche Neuformulierungen gegeneinander abzuwägen sucht: 
Carsten, 24, Referendar:

Also, zuerst/ na ja, die Truppen ziehen ja nicht einfach ab, die werden abgezogen, also kann man hier sagen/ Zuerst muss die Regierung der USA ihre Armee abziehen. Aufgrund eines Befehles, natürlich. Ohne Befehl von oben ist es nicht Abzug sondern Flucht <lacht>. Aber man könnte nicht sagen, Zuerst muss die Regierung der USA den Befehl zum Abzug der Truppen geben, denn nur der Befehl allein reicht nicht, und außerdem kann es ja Wochen dauern, bis die Truppen dann wirklich weg sind, Also gemeint ist, die Truppen müssen weg sein, das verlangt der Friedensvertrag.

Zwei Testpersonen sagen explizit, dass der Abzug der Truppen, wenn damit - wie im zweiten Beispiel, wo im Kontext der Einmarsch vorangeht - das physische Losgehen und Wegmarschieren gemeint sein kann, auf eine Entscheidung zurückgeht, einen Abzug vorzunehmen, womit Abzug zugleich auch die entsprechende Entscheidung durch einen Entscheidungsträger, also einen General oder Politiker, beinhaltet. Nur eine einzige der Testpersonen formulierte diesen Satz so um, dass nur das physische Abziehen der Truppen ausgedrückt wurde. Wie die weiteren Erklärungen zeigen, bedeutet dies aber nicht zwangsläufig, dass sie nicht die Idee der auslösenden Handlung mit einbezogen hat:

\section{Jenny, 31, Übersetzerin}

Die Truppen kommen. Und ziehen wieder ab. In derselben Nacht, wenn der Befehl kom$\mathrm{mt}$, marschieren sie halt weiter. Aber das ist komisch, sowieso, wenn die erst diesen Ort erobern, dass sie gleich wieder abziehen müssen. Na gut, einmarschieren heißt ja nicht erobern, vielleicht marschieren sie ein, sehen, dass es keinen Widerstand gibt und marschieren gleich weiter? Wie war das nochmal? <liest Satz ii erneut] Aha, es heißt nur in den Nachtstunden, nicht, in derselben Nacht. Also das sollte/ das müsste man eh besser formulieren, der Einmarsch und der Abzug erfolgte jeweils in den Nachtstunden. Die sind in der Nacht angekommen, also, vielmehr sind die einmarschiert, und dann kam der Befehl zum Abmarschieren auch nachts. Halt, der Befehl kann auch tags gekommen sein/ Oh je, ich bin heute keine große Hilfe glaube ich. <lacht $>$ Also der Befehl, ist ja unbedingt vorher, aber nur weil das Abmarschieren selbst, dann nachts war, kann ja der General den Befehl auch vorher schon gegeben haben. Oder der General hat den Befehl bekommen und gibt ihn dann weiter. <lacht>

Ganz offensichtlich spielen Präsuppositionen - Soldaten marschieren erst, wenn sie den Befehl dazu bekommen, Truppen bewegen sich nicht selbstständig bzw. eigenverantwortlich, sondern werden durch Befehle dazu gebracht, sich zu bewegen - eine entscheidende Rolle bei der Deutung des Satzes. So gesehen waren die Beispiele von Jäger (1975) vielleicht nicht ideal gewählt, denn vielleicht hätte er selbst die Angelegenheit differenzierter beurteilt, wenn er Kontexte gehabt hätte, in denen klar aufgezeigt wird, dass sich die Truppen tatsächlich (noch) nicht bewegen bzw. noch nicht bewegt wurden. Für einen ergänzenden Einblick waren für die Untersuchung darum - nach einem ersten Prozess der Introspektion und mehreren Probeinterviews - zwei weitere Fälle gewählt worden: iv. Über einen Abzug von Truppen wurde zwar verhandelt, eine abschließende Entscheidung jedoch noch nicht getroffen.

v. Möglicher Truppenabzug aus Afghanistan? Nato befürchtet weitere Alleingänge Trumps (Tagesspiegel 17.6.2020). 
In dem konstruierten Zeitungstitel in Satz iv und dem Zeitungstitel in Satz v wird jeweils deutlicher als in den drei Beispielen von Jäger, dass eine Person die Entscheidung zum Abzug treffen wird bzw. diese treffen könnte, dass es also um eine für die Auslösung der Handlung verantwortliche Entscheidung geht und bei Abzug ,eine Truppenbewegung auslösende strategische Entscheidung' im Vordergrund bzw. am Anfang steht und nicht Abzug als ,physische Umsetzung der Entscheidung. Tatsächlich wurde bei diesen Beispielen von den Informanten und Informantinnen durchgehend die Entscheidung in den Vordergrund gerückt, wie die folgenden Antworten illustrieren sollen:

\section{Kerstin, 38, Journalistin}

Also das liest sich wie eine Zeitungsmeldung, aber ich lasse das mit der Frage hier im Titel/ Das ist doch sicher eine Überschrift von einem Artikel über Trump! Aber ich lasse das mal unkommentiert. Das ist furchtbar, wie in der Bildzeitung. Also, möglicher Truppenabzug, wieder ein Abzug, und wieder geht es um Truppen, die irgendwo sind, wo sie nicht hingehören. Also, meiner Meinung nach. Und möglich, warum möglich? Wahrscheinlich hat Trump was getweetet und darum ist es möglich. Aber den Befehl gibt es noch nicht, sonst wäre es ja sicher, dann wäre der Abzug beschlossene Sache, wäre zu erwarten. Also haben wir bestimmt nur den Tweet, aber die Befehle kennen wir nicht. Ich würde wohl schreiben: Nato befürchtet Abzug der US-Truppen als erneuten Alleingang von Trump.

Vera, 62, Deutschlehrerin

Hier haben wir jetzt wirklich Trump. Aber, hier ist es ganz klar, der Marschbefehl wurde noch gar nicht gegeben.

Helga, 77, pensionierte Deutschlehrerin

Eine abschließende Entscheidung/ betrifft das wieder den Trump? Ja eigentlich ist das gut formuliert. Eine endgültige/ Lösung? Lösung geht nicht, klingt wie Endlösung/ da fällt mir jetzt nichts ein. Ich finde der Bezug ist falsch, der Abzug ist immer möglich, aber das ist natürlich die Verkürzung. Welcher Truppen? Der amerikanischen Truppen? Abschließende Entscheidung? Also der Befehl? [...] Statt Alleingang vielleicht einsame Entscheidungen Trumps. <lacht $>$ Das ist zu leger, aber so sagt man es, wenn jemand etwas ohne Absprachen macht, und das ist ja hier der Fall, wenn er das ohne Rücksprache mit der Nato entscheidet. Sonst fällt mir da nichts ein, Truppenabzug ist Truppenabzug.

Ganz offensichtlich ist Jäger als Sprecher des Deutschen und des Tschechischen, und als Experte für Übersetzungen zwischen diesen beiden Sprachen, nicht der ideale Informant für die Frage, wie deutsche Muttersprachler und Muttersprachlerinnen den Ausdruck Abzug in den von ihm genannten Konstruktionen verstehen, denn er sieht die Sätze anders als unsere Testpersonen. Anzunehmen ist, dass Jäger die jeweils verstandenen Bedeutungen und damit die für die Deutschmuttersprachler und -muttersprachlerinnen unserer Perzeptionsstudien nicht sofort ersichtliche bzw. nicht als solche „empfundene“ Mehrdeutigkeit ja auch oder gerade wegen des Abgleichs mit den tschechischen Übersetzungslösungen ermittelt.

Im Deutschen ist Abzug zwar im Sinne der Erläuterungen Jägers durch den Bezug auf verschiedene mögliche dem deverbalen Substantiv zugrundeliegenden Verben abziehen und jmd. abziehen polysem. Die Sprecher und Sprecherinnen scheinen diese inhärente Polysemie jedoch nicht zu aktualisieren, da dem von abziehen hergeleiteten Abzug ja das von jmd. abziehen hergeleitete Abzug als Entscheidung immer vorausgehen muss bzw. Abzug als auslösende Handlung erst abgeschlossen ist, wenn es zum Abzug als ausfüh- 
rende Handlung gekommen ist (der eine Abzug den anderen also umfasst). Die von Jäger aufgrund der Kenntnis des Tschechischen bemerkte Polysemie ist also gewissermaßen nur im Hinblick auf die Morphologie anzunehmen, wird aber von den Muttersprachlern und Muttersprachlerinnen eigentlich gar nicht so realisiert, da die beiden Einzelhandlungen (a) Abzug der Truppen auslösender Befehl und (b) den Abzugsbefehl umsetzende Bewegung der Truppen nicht separiert werden, da (b) ohne (a) gar nicht zustanden kommt. Nur wenn (a) noch nicht realisiert wurde bzw. nur wenn (a) als Möglichkeit in der Zukunft liegt, wird diese Einbettung von (b) in (a) notorisch.

Auch wenn Jäger mit seiner Sicht nicht unbedingt recht hatte, so ist ihm doch beizupflichten, dass man beachten muss, wie etwas jeweils verstanden wird - und das betrifft ganz deutlich die Empfängerseite und damit letztlich auch den Zieltext. Ihm ist aufgrund der unterschiedlichen Übersetzungen im Tschechischen aufgefallen, dass es im Deutschen mehrere Lesarten gibt. Er fehlinterpretiert jedoch - im Vergleich zu den Informanten und Informantinnen - die im Deutschen offenbar vorherrschende Interpretation des Satzes, denn in allen Kontexten ist im Deutschen eigentlich immer klar, dass der Abzug auf einen Befehl von oben erfolgt und es um eben diesen Befehl als Auslöser geht, und nicht um die Truppenbewegungen aus eigenem Antrieb heraus, also nicht um ein dem intransitiven Verb abziehen zugehöriges bzw. von ihm abgeleitetes Substantiv Abzug. Das bedeutet, dass sich für Jäger erst über die Übersetzung im Tschechischen die Mehrdeutigkeit von Abzug als Übersetzungsproblem herauskristallisiert, da er davon ausgeht, dass eine Entscheidung getroffen werden müsse, welche der beiden möglichen Lesarten ins Tschechische übertragen werden sollte.

Das eigentliche Problem ist also hier die Frage danach, wie die aus dem Deutschen ins Tschechische übersetzenden Personen den deutschen Satz jeweils verstanden haben, was also ihre Beweggründe für eine Entscheidung für den Gebrauch von stažení oder von odchod gewesen sind, denn der Abzug im Sinne des intransitiven Verbes abziehen wäre ja eigentlich eine zu kurz greifende Fehlinterpretation, da es auf alle Fälle um den Befehl von oben (und die Vollendung seiner Umsetzung), also den Bezug zu einem intransitiven jmd. abziehen geht.

In der theoretischen Auseinandersetzung mit derartigen strukturellen Entscheidungen ist natürlich stets zu berücksichtigen, dass es durchaus eine Divergenz geben kann zwischen dem, was ein Individuum beispielsweise in einem schriftlichen Text ausdrücken wollte, und dem, wie es von der Mehrheit der Leser und Leserinnen verstanden wird.

Ein Beispiel im Deutschen wäre hier die unterschiedliche Bedeutung von anscheinend ,wie es scheint, dem Anschein nach, offenbar' (Hauptbedeutung neben dem laut Duden nur seltenen, dem Anschein nach gegeben, vorhanden, bestehend') und scheinbar, aufgrund einer Täuschung wirklich, als Tatsache erscheinend, aber in Wahrheit nicht wirklich gegeben. Eine Minderheit der Deutschsprachigen verwendet scheinbar im Sinne von anscheinend, und wenn aus dem jeweiligen Kontext nicht ganz eindeutig hervorgeht, wie das Element zu verstehen ist, zeigt sich schnell, dass die Mehrheit der Informanten und Informantinnen, ungeachtet der Intention des Autors bzw. der Autorin, die im Duden nicht als selten markierte Bedeutung aktualisiert. Wir können uns in der gesprochenen wie der geschriebenen Sprache jedoch oft nicht sicher sein, wie es nun tatsächlich gemeint war, das heißt, ohne einen die Aussage klärenden Kontext können wir nicht sicher sein, welche Bedeutung beabsichtigt war. 
Ein anderes Beispiel aus der Werbung zeigt deutlich, wie der Kontext bei der Divergenz zwischen dem Gemeinten und dem Gesagten die Interpretation stützen bzw. überhaupt erst ermöglichen kann. In der Aussage „Mit unserer ferngesteuerten Klimaautomatik brauchen Sie in Ihrem Fahrzeug nie mehr [zu] frieren. Sie können die Standheizung (falls vorhanden) so programmieren, dass Ihr Volvo angenehm warm und die Scheiben enteist sind, sobald Sie einsteigen" (Volvo 2020) wird eigentlich sofort klar, dass es statt sobald hier wenn heißen müsste bzw. dass sobald hier entgegen der eigentlichen Bedeutung dieser Konjunktion mit der Bedeutung ,wenn' verwendet worden sein muss, denn vom Ablauf her ist auch bei wenig ausgeprägtem technischen Verständnis offensichtlich, dass der Wagen mit der beschriebenen Funktion die Scheiben nach und nach vorheizt und diese dann spätestens zur eingestellten Zeit enteist sind und nicht etwa in dem Augenblick, genau in dem Moment, in dem man einsteigt, die Scheiben schlagartig enteist werden.

Wesentlich erscheint mir hier ganz zuvorderst der Umstand, dass Jäger hier über das möglicherweise divergierende Verständnis der deutschen Struktur und die Relevanz der jeweiligen Perzeption durch die übersetzende Person für die Übersetzungsentscheidung spricht. Nachrangig ist der Umstand, dass ihm nicht auffällt, dass sowohl seine eigene Sicht auf die Dinge als auch die Erklärung für die jeweiligen Übersetzungsentscheidungen im Tschechischen mit den individuellen Sichtweisen auf den Text zu erklären sind, also das Ergebnis einer idiolektal geprägten, individuellen Lektüre sind.

Damit ist Jäger der in den 1970er Jahren noch immer dominierenden generativistischen Position des absoluten Vertrauens in das eigene Sprecherwissen, die eigene sprachliche Intuition, die Introspektion verhaftet. Er begeht einen aus moderner Sicht zwar bedauerlichen, aber zeitgemäßen Denkfehler, denn in den 1970er Jahren wurden die Positionen der (eigentlich absurden) generativistischen Herangehensweise, das eigene Urteil zu verallgemeinern, praktisch nicht in Frage gestellt.

Aber sein Hinweis auf die Möglichkeit unterschiedlicher Lesarten und somit auf ein unterschiedliches Verständnis ein und derselben Struktur bei unterschiedlichen Rezipienten bzw. Rezipientinnen ist ein klarer Hinweis auf das Bewusstsein um die Relevanz der Berücksichtigung der individuellen Sicht auf die Dinge zum Verständnis vom Übersetzungsentscheidungen. Jäger ging es um Polysemie als Übersetzungsproblem, und die beabsichtigte Klassifizierung unterschiedlicher semantischer Konstellationen und ihrer Auswirkungen auf die Übersetzung nahm er, als Übersetzungswissenschaftler, eben aus der übersetzungsrelevanten Analyse von Übersetzungen vor. Stolperstein ist hier, dass er noch in dem Glauben verhaftet ist, dass seine individuelle Sicht (also die Sicht eines mehrsprachigen, auf Übersetzung spezialisierten Akademikers, Linguisten und Übersetzungswissenschaftlers) ausreichend sein könnte.

Im Kern betrachtet Jäger hier das Problem der Perzeption, und er geht immerhin so weit, die Perspektive der tschechischen Übersetzer oder Übersetzerinnen mit der eigenen Sicht als Deutschmuttersprachler abzugleichen. Die Berücksichtigung der Perzeption, und wie hier im Kontrast zwischen der Perzeption durch mehrere Muttersprachler bzw. Muttersprachlerinnen der Zielsprache gegenüber der Perzeption durch einen Muttersprachler der Ausgangssprache, ist fortschrittlich und für ihre Zeit bemerkenswert. Aus heutiger Sicht wird klar, dass diese Überlegungen unbedingt hätten ausgearbeitet werden müssen, dass sie Ausgangspunkt für eine vertiefte Auseinandersetzung mit der Frage der Perzeption und der Wirkung hätten sein können. 
Wir sehen hier, dass die Leipziger Schule schon sehr früh innovative Ideen hatte und neue Wege ging, die jetzt, mit neuen Möglichkeiten der Arbeit mit riesigen Datenmengen, mit Onlineerhebungen in großem Stil und mit relativ geringem Kostenaufwand, fortgeführt werden können. Doch trotz der frühen Hinweise auf die Bedeutung der Wirkung von Ausgangs- und Zieltext, wie seit den 1960er Jahren in den Texten der Leipziger Schule, ist die Berücksichtigung der Perzeption von einzelnen Ausdrücken, Konstruktionen oder ganzen Texten, idealerweise sogar in Ausgangs- und Zielsprache, bis heute noch immer eine Ausnahme in translatologischen Studien (vgl. Sinner 2014).

\section{Schluss und Ausblick}

Das vorgestellte Beispiel von Jäger (1975) ist in mehrerlei Hinsicht bemerkenswert. Es finden sich in diesem Text einerseits Aussagen, die für aktuelle Probleme der Übersetzungswissenschaft im Hinblick auf den Umgang mit Wirkung von Bedeutung sind. Andererseits spricht es Bände, dass man Ausführungen aus einer vor über vier Jahrzehnten veröffentlichten wissenschaftlichen Arbeit im Jahre 2020 verwenden kann, nicht etwa, um in der Vergangenheit nach Aspekten zu suchen, die für das Verständnis der Disziplingeschichte von Nutzen sein können, sondern weil man die Betrachtung des von Jäger (1975) behandelten Textauszugs direkt mit modernen Fragestellungen verknüpfen kann. Bemerkenswert ist aber auch, dass es bei der Untersuchung aktueller Fragen und der Bewältigung moderner Probleme helfen kann, in der eigenen Disziplin Jahrzehnte zurückzugehen zu Texten, die aus Sicht mancher der Leipziger Schule weniger gut gesonnener Autoren und Autorinnen als „überwunden“ geltende Positionen vertreten.

Dass die Leipziger Schule große Innovationskraft hatte, mag kaum jemand abstreiten, aber dass manche der vor Jahrzehnten verfassten Texte bis heute brandaktuell sein würden, dass manche Detailbetrachtungen Jahrzehnte später kommende „wissenschaftliche Trends" thematisch vorwegnahmen, ist beachtlich. Heute ist man eher bereit zuzugeben, dass die Leipziger Schule eine Vorreiterrolle hatte, schon in ihren Anfängen sehr modern war und vor allem die untersuchten Themen in einer Art und Weise behandelte, die auch heute noch erstaunen lässt. An einem Beispiel für die Übersetzung zwischen Tschechisch und Deutsch konnte hier gezeigt werden, dass die Überlegungen von Gert Jäger zum Problem der Synonymie in der Translation einen aus heutiger Sicht bedeutenden Aspekt ansprach und für die Untersuchung der Rolle des individuellen Textverständnisses und der Perzeption von Texten einen wertvollen Anknüpfungspunkt darstellt.

Über die Präsenz des Tschechischen in der Ausbildung in Leipzig ist eingangs bereits gesprochen worden. Es erscheint mir jedoch bedeutsam, erneut darauf hinzuweisen, welch große Rolle das Tschechische tatsächlich spielte.

Es ist zwar (natürlich) für das wissenschaftliche Ergebnis nicht unbedingt relevant, aus welcher Sprache Beispiele stammen, hervorhebenswert ist in diesem Zusammenhang meines Erachtens aber, dass hier der Vergleich mit tschechischen Lösungen (und nicht wie so oft mit englischsprachigen Beispielen) dazu genutzt werden konnte, ein Problem der allgemeinen Translatologie bzw. ein allgemeines übersetzungstheoretisches Problem darzulegen, ohne dass bei der Mehrheit der Lesenden die Kenntnisse dieser Sprache vorausgesetzt werden konnten bzw. können. Dies zeigt, dass tschechische Beispiele genauso 
gut funktioniert haben wie irgendwelche aus dem vermeintlich so viel internationaleren Englisch, wo man in der Regel auch die Erklärungen mitbekommt, ja meist mit angegeben bekommen muss, was genau die einzelnen Sätze nun jeweils bedeuten.

In Zeiten, in denen in der Translatologie ebenso wie in der Sprachwissenschaft das Englische als Veröffentlichungssprache und als Objektsprache übermächtig ist und in denen ein Großteil der Untersuchungen das Englische entweder als eine der beiden Objektsprachen direkt einbezieht oder aber auf Grundlage von Arbeiten entsteht, in denen das Englische eine solche zentrale Rolle einnimmt, ist es eine Befriedigung festzustellen, dass man mit tschechischen Beispielen ebenso gut arbeiten und diese auch ohne tiefer gehende Kenntnisse slawischer Sprachen mit Gewinn lesen kann. Dies möchte ich auch als Ansporn verstehen, nicht um der vermeintlich so viel besseren internationalen Rezeption willen unvermeidlich mit dem Englischen zu arbeiten, Beispiele aus der englischen Sprache "exemplarisch" für alle Sprachen der Welt heranzuziehen und aus Sprachpaaren, von denen eine der beiden Sprachen unvermeidlich Englisch ist, auf die Übersetzungsbeziehungen beispielsweise zwischen zwei sehr selten übersetzten Sprachen schließen zu wollen. Dies hat besondere Bedeutung in Zeiten, wo Sätze wie das Chomsky-Zitat „Take any language, for example, English“ bereits als Running Gag zur Belustigung über die fehlende Empirie in generativistischen bzw. stark formalisierenden Studien angesehen werden (s. Menz 2016, Linguisten.de 2019).

Angesichts der großen Relevanz der Auseinandersetzung mit der Translation im deutsch-tschechischen Sprachpaar erscheint eine wissenschaftliche und historiographische Aufarbeitung der Beiträge der Leipziger Schule zum hier betrachteten Sprachenpaar wünschenswert, idealerweise einhergehend mit einer Herausgabe einer Anthologie der zentralen Texte in deutscher wie tschechischer Sprache sowie in anderen international besser rezipierten Sprachen wie Englisch, Französisch und Spanisch. Insbesondere könnten dadurch auch inhaltliche Analysen angestoßen werden, ob beispielsweise in den frühen Publikationen in tschechischer Sprache Aspekte konstatierbar sind, die in Veröffentlichungen in anderen Sprachen nicht berücksichtigt oder anders dargestellt sind. Da, wie gesehen, die slawisch-deutschen Sprachpaare eine privilegierte Rolle in der Herausbildung und dem Tätigkeitsbereich der Leipziger Schule einnahmen, sollten diese Texte, so wie die deutschsprachigen Schlüsseltexte der Leipziger Schule in Editionen in portugiesischer und spanischer Sprache (s. Cardozo et al. 2008, Jung et al. 2013) erschienen sind, mit Stolz international in Übersetzungen in unterschiedliche Sprachen zugänglich gemacht werden. Damit könnte nicht nur der in der Rezeptionskritik genannte Nachteil für die internationale Wahrnehmung ausgeglichen werden, dass wesentliche Schriften in wenig zugänglichen Sprachen veröffentlicht wurden, sondern durch die gesammelte Herausgabe in international besser zugänglichen Verlagen oder in Onlinepublikationen der Zugang zu vielen Publikationen, die außerhalb der Bibliotheken in einigen Ländern wie Polen, Tschechien oder Russland kaum erhältlich sind, ermöglicht werden.

Einen Beitrag zur Historiographie der Rolle des Tschechischen in der Leipziger Schule bzw. in der Translationsausbildung in Leipzig leistet derzeit bereits das 2013 initiierte Oral-History-Projekt zum Dolmetschen bzw. zur Translation allgemein am IALT (s. Sinner 2020); Scholz (2020) beispielsweise untersucht in ihrer M.A.-Arbeit im Rahmen dieses Projekts unter anderem auf Grundlage des Universitätsarchivs der Universität Leipzig die Entwicklung der Ausbildung im Bereich Sprachmittlung für Tschechisch. Mithilfe 
der so erstellten Jahrgangsübersichten über die erfolgten Immatrikulationen sollen nun die Kriterien für die Zusammensetzung der Gruppe der zu befragenden Lehrkräfte und Absolventen und Absolventinnen im Bereich Tschechisch bestimmt werden und die entsprechenden Personen für Oral-History-Interviews ermittelt und befragt werden.

\section{BIBLIOGRAPHIE}

Auroux, Sylvain: Science et temporalité, in: ed. Daniele Gambarara - Stefano Gensini -Benešová, Rút: Otto Kade a jeho prínos translatologii. Diplomarbeit. Praha: Univerzita Karlova 2019. Online unter: https://is.cuni.cz/webapps/zzp/detail/203055/ [23. 11.2020].

Bernardo, Ana Maria Garcia: A tradutologia contemporânea. Tendências e perspectivas no espaço de língua alemã, Lisboa: Fundação Calouste Gulbekian 2009.

Bochmann, Klaus: Theorie und Methoden der Sprachpolitik und ihrer Analyse, in: ed. Bochmann, Klaus et al.: Sprachpolitik in der Romania. Zur Geschichte sprachpolitischen Denkens und Handelns von der Französischen Revolution bis zur Gegenwart, Berlin/New York: de Gruyter 1993, S. 3-62.

Bolaños Cuéllar, Sergio: Towards an Integrated Translation Approach. Proposal of a Dynamic Translation Model (DTM), Hamburg: Universität Hamburg 2008. Online unter: http://ediss.sub.uni-hamburg.de /volltexte/2008/3726/pdf/DissertationSBolanos2008.pdf [25. 8. 2015].

Busse, Dietrich - Teubert, Wolfgang: Ist Diskurs ein sprachwissenschaftliches Objekt? Zur Methodenfrage der historischen Semantik, in: ed. Dietrich Busse - Fritz Hermanns - Wolfgang Teubert: Begriffsgeschichte und Diskursgeschichte. Methodenfragen und Forschungsergebnisse der historischen Semantik, Opladen: Westdeutscher Verlag 1994, S. 10-28.

Cañuelo Sarrión, Susana - Hernández Socas, Elia - Ivanova, Vesella: La recepción de la Escuela traductológica de Leipzig en España, in: ed. José Juan Batista Rodríguez - Carsten Sinner - Gerd Wotjak: La escuela traductológica de Leipzig: Continuación y recepción, Frankfurt am Main u.a.: Lang 2019, S. 55-92.

Cardozo, Maurício - Heidermann, Werder - Weininger, Markus J.: A Escola Tradutológica de Leipzig, Frankfurt am Main u.a.: Lang 2008.

Castro Yáñez, Ginette: Rezension von La Escuela traductológica de Leipzig. Sus inicios, su credo y su florecer (1965-1985) herausgegeben von Gerd Wotjak, Carsten Sinner und José Juan Batista Rodríguez, Frankfurt am Main u.a.: Lang 2013, Infoling 3.34, S. 1-20. Online unter: http://infoling.org/informacion/Review207.html [3. 8. 2015].

de la Cruz Recio, Manuel: Der kommunikative Wert in der Leipziger Schule: eine Revision, in: ed. Holger Siever: Translationswissenschaft in Spanien, München: Akademische Verlagsgemeinschaft München 2016, S. 275-293.

Didaoui, Mohammed: Qualitätslektorat, in: ed. Snell-Hornby, Mary et al.: Handbuch Translation, Tübingen: Stauffenburg 2006, S. 381-383.

Dittmar, Norbert: Grundlagen der Soziolinguistik. Ein Arbeitsbuch mit Aufgaben, Tübingen: Niemeyer 1997.

Haßler, Gerda: Les séries de textes dans l'histoire de la linguistique. in: ed. Annick Englebert - Michel Pierrard - Laurence Rosier - Dan Van Raemdonck: Actes du xxıIe Congrès International de Linguistique et Philologie Romanes. Bruyelles, 23-29 juillet 1998. Vol. I: L'histoire de la linguistique, médiatrice de théories, Tübingen: Niemeyer 2000, S. 97-104.

Guespin, Louis: Soziabilitätsstruktur und Sprachgebrauch in ihren Wechselbeziehungen, Linguistische Arbeitsberichte 51, 1985, S. 26-41.

Haßler, Gerda: Textos de referencia y conceptos en las teorías lingüística de los siglos XVII y XVIII, in: ed. Miguel Ángel Esparza Torres - Benigno Fernández Salgado - Hans-Josef Niederehe: SEHL 2001 Estudios de Historiografía Lingüística. Actas del III Congreso Internacional de la Sociedad Española de Historiografía Lingüística. Vigo, 7-10 de febrero de 2001, Hamburg: Buske 2002, S. 559-586.

Hrdinová, Eva Maria: Von drei translatorischen Nüssen des Aschenbrödels, Zeitschrift für Slawistik 63, 2, 2018, S. 338-350. 
Hrdinová, Eva Maria: Die Übersetzung der Propria in Märchen im Kontext der Realienübersetzung, in: ed. Christian Bahr - Carsten Sinner: Onomastik und Übersetzung, Zahna: Baar-Verlag, im Druck.

Jäger, Gert: Die sprachlichen Bedeutungen - das zentrale Problem bei der Translation und ihrer wissenschaftlichen Beschreibung, in: ed. Gert Jäger - Albrecht Neubert: Bedeutung und Translation, Leipzig: Verlag Enzyklopädie 1986, S. 5-66.

Jäger, Gert: Translation und Translationslinguistik, Halle (Saale): Max Niemeyer 1975.

Levý, Jiř́i: Umění prekladu, Praha: Československý spisovatel 1963.

Levý, Jiř́: Die literarische Übersetzung: Theorie einer Kunstgattung, Frankfurt am Main / Bonn, Athenäum 1969.

Jung, Linus: La escuela traductológica de Leipzig, Granada: Comares 2000.

Jung, Linus - Sinner, Carsten - Batista Rodríguez, José Juan: De las tinieblas a la luz: los presupuestos teóricos de la Escuela de Leipzig en lengua española, in: ed. Gerd Wotjak - Carsten Sinner - Linus Jung - José Juan Batista Rodríguez: La Escuela traductológica de Leipzig. Sus inicios, su credo y su florecer (1965-1985), Frankfurt am Main u.a.: Lang 2013, S. 1-20.

Kade, Otto: Aufgaben der Übersetzungswissenschaft. Zur Frage der Gesetzmäßigkeit im Übersetzungsprozess, Fremdsprachen 2, 1963, S. 83-94.

Kade, Otto: Zufall und Gesetzmäßigkeit in der Übersetzung, Leipzig: Verlag Enzyklopädie 1968.

Kade, Otto: Die Sprachmittlung als gesellschaftliche Erscheinung und Gegenstand wissenschaftlicher Untersuchung, Leipzig: Verlag Enzyklopädie 1980.

Knapp, Karlfried - Knapp-Potthoff, Annelie: Sprachmittlertätigkeit in interkultureller Kommunikation, in: ed. Jochen Rehbein: Interkulturelle Kommunikation, Tübingen: Narr 1985, S. 450-463.

Lerman, Margarita: La causa justa de los pueblos árabes - „Die israelische Frage“ aus kubanischer Perspektive 1965 bis 1973. Diplomarbeit, Universität Leipzig 2019.

Take any language, for Example English... Online unter: https://blog.linguisten.de/post/187881922690 /take-any-language-for-example-english [2.6.2020].

Lutz, Anke: Das Dolmetscher-Institut an der Handelshochschule, in: ed. Hans Göschel: Die Handelshochschule in Leipzig, Leipzig: Handelshochschule Leipzig 2008, S. 130-134.

Lutz, Anke: Auszug aus Dissertation: Zur Integration des Sachfachs in translatorischen Studiengängen. Historische Retrospektive - Empirische Analyse - Curriculares Modell. Leipzig: Institut für Angewandte Linguistik und Translatologie, unveröffentlichtes Manuskript, ohne Jahr.

Einführung in die Soziolinguistik. Online unter: https://vowi.fsinf.at/wiki/Uni_Wien:Einführung_in_die _Soziolinguistik_VO_(Menz)/Mitschrift [2. 6. 2020].

Neubert, Albrecht: Regeln des Übersetzens, Fremdsprachen 2, 1965, S. 83-89.

Neubert, Albrecht: Pragmatische Aspekte der Übersetzung, in: ed. Albrecht Neubert: Grundfragen der Übersetzungswissenschaft, Leipzig: Enzyklopädie 1968, S. 21-33.

Oksaar, Els: Idiolekt, in: ed. Ulrich Ammon - Norbert Dietmar - Klaus J. Mattheier: Sociolinguistics. An International Handbook of the Science of Language and Society, Berlin: de Gruyter 1987, S. 293-297.

Oksaar, Els: Idiolekt als Grundlage der variationsorientierten Linguistik, Sociolinguistica 14, 2000, S. 37-42.

Pennisi, Antonino: Language Philosophies and the Language Sciences. A Historical Perspective in Honour of Lia Formigari, Münster: Nodus 1996, S. 27-32.

Prunč, Erich: Entwicklungslinien der Translationswissenschaft. Von den Asymmetrien der Sprachen zu den Asymmetrien der Macht, Berlin: Frank \& Timme 2007.

Pym, Anthony: Teorías contemporáneas de la traducción, La Habana: Editorial Arte y Literatura 2014.

Salevsky, Heidemarie: In Memoriam Otto Kade (28. 3. 1927 - 2. 11. 1980), in: ed. Heidemarie Salevsky: Wissenschaftliche Grundlagen der Sprachmittlung. Berliner Beiträge zur Übersetzungswissenschaft, Frankfurt am Main u.a.: Lang 1992, S. 11-15.

Schmidt, Heide: Kommentar zum Äquivalenzverständnis, Fremdsprachen 4, 1987, S. 249-255.

Scholz, Marie: Ausbildungsstatistiken und Interviews - Ein Beitrag zum Projekt Oral History des Dolmetschens. Diplomarbeit. Universität Leipzig 2020.

Sinner, Carsten: El castellano de Cataluña. Estudio empírico de aspectos léxicos, morfosintáticos, pragmáticos y metalingüisticos, Tübingen: Niemeyer 2004.

Sinner, Carsten: Varietätenlinguistik. Eine Einführung, Tübingen: Narr 2014. 
Sinner, Carsten: Leipzig School revisited. Kade, Neubert, and Jäger seen from a 21st century perspective, in: ed. Julia Richter - Cornelia Zwischenberger - Stefanie Kremmel - Karlheinz Spitzl: (Neu-)Kompositionen. Aspekte transkultureller Translationswissenschaft. Liber amicorum für Larisa Schippel, Berlin: Frank \& Timme 2016, S. 131-150.

Sinner, Carsten: „Translatología perceptiva. La percepción como reto“, in: ed. Ingrid Cáceres Würsig Carmen Gómez García - Lorena Silos: Realitäten, Herausforderungen und Reflexionen. Ein interdisziplinärer Blick auf die zeitgenössische Germanistik, Berlin: Frank \& Timme 2019, S. 155-187.

Sinner, Carsten: On Oral History in Translation and Interpretation Studies, chronotopos - A Journal of Translation History 2, 1\&2, im Druck.

Sinner, Carsten - Morales Tejada, Beatriz: Translatorische Perzeptionsstudien als Grundlage der Bestimmung gelungener Übersetzungen, Lebende Sprachen 60, 1, 2015, S. 111-123.

Sinner, Carsten - Wieland, Katharina: Eine translationswissenschaftliche Sicht auf Sprachmittlung im Fremdsprachenunterricht, in: ed. Daniel Reimann - Andrea Rössler: Sprachmittlung im Fremdsprachenunterricht, Tübingen: Narr 2013, S. 93-113.

Snell-Hornby, Mary: A forgotten Pioneer? The legacy of Otto Kade in Translation Studies today, in: ed. Gerd Wotjak: Quo vadis Translatologie? Ein halbes Jahrhundert universitäre Ausbildung von Dolmetschern und Übersetzern in Leipzig, Berlin: Frank \& Timme 2007, S. 387-395.

Stolze, Radegundis: Übersetzunsgtheorien. Eine Einführung. Tübingen: Narr 1994.

Volvo on call. Kontrolle und Komfort. Online unter: www.volvocars.com/de/volvo/innovationen/konnektivitaet/volvo-on-call [17. 7. 2020].

Wotjak, Gerd (ed.): 50 Jahre Leipziger Übersetzungswissenschaftliche Schule. Eine Rückschau anhand von ausgewählten Schriften und Textpassagen, Frankfurt am Main u.a.: Lang 2006.

Wotjak, Gerd: Die Leipziger Übersetzungswissenschaftliche Schule. Anmerkungen eines Zeitzeugen“, in: ed. Lew N. Zybatov: Translation zwischen Theorie und Praxis, Innsbrucker Ringvorlesungen zur Translationswissenschaft I, Frankfurt am Main u.a.: Lang 2002, S. 87-119. Online unter: http://www .unileipzig.de/ ialt/cms/uploads/leipzigerschule.pdf [3. 8. 2015].

Wotjak, Gerd: La Escuela de Traductología de Leipzig: su nacimiento, sus representantes más destacados y sus postulados (testimonio de un colaborador no directanmente involucrado), Hieronymus complutensis 9-10, 2002-2003, S. 7-26.

Wotjak, Gerd - Sinner, Carsten - Jung, Linus - Batista Rodríguez, José Juan: La Escuela traductológica de Leipzig. Sus inicios, su credo y su florecer (1965-1985), Frankfurt am Main u.a.: Lang 2013.

\section{RESUMEN}

Después de una visión global de la historia de la formación de traductores e intérpretes en Leipzig y de la Escuela traductológica de Leipzig, el artículo debate primero las lagunas y deficiencias en la recepción de la Escuela de Leipzig para luego mostrar que las críticas a menudo eran injustificadas y se debían a una falta de conocimiento o comprensión de los textos en cuestión. Después, se analiza la comprensión de Sprachmittlung 'mediación lingüística' y Translation 'traslación'; una perspectiva terminológica y conceptual revela la aparentemente malinterpretación de los textos clave de la escuela de Leipzig por parte de muchos de los autores que la criticaron. Por último, se ejecuta un examen ejemplar de uno de los textos claves (Jäger 1975) que permite poner de manifiesto la gran capacidad innovadora de la Escuela de Leipzig.

Prof. Dr. Carsten Sinner

Institut für Angewandte Linguistik und Translatologie, Universität Leipzig sinner@rz.uni-leipzig.de 